\title{
Allen-Cahn approximation of mean curvature flow in Riemannian manifolds I, uniform estimates
}

\author{
AdRIANO PisAnte AND FABIO PUNZO
}

\begin{abstract}
We are concerned with solutions to the parabolic Allen-Cahn equation in Riemannian manifolds. For a general class of initial conditions we show nonpositivity of the limiting energy discrepancy. This in turn allows us to prove an almost monotonicity formula (a weak counterpart of Huisken's monotonicity formula) which gives a local uniform control of the energy densities at small scales.

These results will be used in [41] to extend previous important results from [31] in Euclidean space, showing convergence of solutions to the parabolic AllenCahn equations to Brakke's motion by mean curvature in Riemannian manifolds.
\end{abstract}

Mathematics Subject Classification (2010): 53C44 (primary); 35B25, 35B45 (secondary).

\section{Introduction}

We are concerned with the Allen-Cahn equation

$$
\partial_{t} u^{\varepsilon}=\Delta u^{\varepsilon}-\frac{1}{\varepsilon^{2}} f\left(u^{\varepsilon}\right) \text { in } M \times(0, \infty),
$$

completed with the initial condition

$$
u^{\varepsilon}=u_{0}^{\varepsilon} \quad \text { in } M \times\{0\}
$$

Here $\varepsilon>0$ is a small parameter, $M$ is an $N$-dimensional Riemannian manifold with Ricci curvature bounded from below, $\Delta$ is the Laplace-Beltrami operator on $M$, the function $f$ is the derivative of a potential $F$ with two wells of equal depth at

Received August 13, 2013; accepted in revised form April 24, 2014.

Published online February 2016. 
$u=-1$ and at $u=1$. To be specific, we will always assume for simplicity that $f$ satisfies

(i) $f=F^{\prime}$, with $F \in C^{\infty}(\mathbb{R}), F$ even;

(ii) $f(0)=f( \pm 1)=0, f<0$ in $(0,1), f>0$ in $(1, \infty)$,

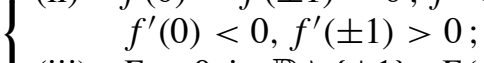

(iii) $F>0$ in $\mathbb{R} \backslash\{ \pm 1\}, F( \pm 1)=0$;

(iv) $\min _{[\alpha, \infty)} F^{\prime \prime}>0$, for some $\alpha \in(0,1)$.

A typical example is

$$
F(u)=\frac{1}{2}\left(1-u^{2}\right)^{2}, \quad f(u)=2 u\left(u^{2}-1\right) .
$$

We set

$$
f_{\varepsilon}(u):=\frac{1}{\varepsilon^{2}} f(u), \quad F_{\varepsilon}(u):=\frac{1}{\varepsilon^{2}} F(u) .
$$

Observe that problem

$$
\begin{gathered}
\partial_{t} u^{\varepsilon}=\Delta u^{\varepsilon}-\frac{1}{\varepsilon^{2}} f\left(u^{\varepsilon}\right) \quad \text { in } \mathbb{R}^{N} \times(0, \infty), \\
u^{\varepsilon}=u_{0}^{\varepsilon} \quad \text { in } \mathbb{R}^{N} \times\{0\},
\end{gathered}
$$

which corresponds to problem (1.1)-(1.2) in the special case $M=\mathbb{R}^{N}$, has been the object of detailed investigations in order to describe formation and evolution of interfaces (approximatively) driven by their mean curvature. Indeed, it is wellknown that the term $f_{\varepsilon}$ forces the solution $u^{\varepsilon}$ to problem (1.3)-(1.4) to problem to take values 1 or -1 , as $\varepsilon \rightarrow 0^{+}$; moreover, the interface that separates the two regions of $\mathbb{R}^{N} \times(0, \infty)$ in which $u^{\varepsilon}$ converges to 1 or -1 , say the region where $\left\{\left|u^{\varepsilon}\right|<\frac{1}{2}\right\}$, is a set of thickness of order $\varepsilon$ that in the limit as $\varepsilon \rightarrow 0$ approximatively moves by mean curvature flow as long as time varies. A large number of papers have been devoted to this type of results, using several methods. Without any claim for completeness, we mention [1,3,8-15,23-25,31,38,42,43]; observe that also similar questions have been addressed also for the stationary equation (see, e.g., $[29,36,39,40])$ and for systems (see, e.g., [6,33]). For a comprehensive account of literature on this subject, also containing the description of main results obtained and various methods used, we refer the reader to [43] and references therein. In this connection, note that in the literature several notions of mean curvature flows have been considered (see, e.g., $[4,5,13,19-23,32,37,43]$ ).

In the sequel, before describing the results of the present paper, we limit ourselves to recalling those established in [31]; moreover, in general, we shall briefly explain the line of arguments followed there to obtain them.

An important role is played by the one-dimensional standing wave $q^{\varepsilon}$ for (1.3), for which there hold:

$$
\begin{aligned}
& q_{r r}^{\varepsilon}(r)-f_{\varepsilon}\left(q^{\varepsilon}(r)\right)=0, \quad r \in \mathbb{R}, \\
& q_{r}^{\varepsilon}>0, q^{\varepsilon}(0)=0, \quad \lim _{r \rightarrow+\infty} q^{\varepsilon}(r)=1, \quad \lim _{r \rightarrow-\infty} q^{\varepsilon}(r)=-1 .
\end{aligned}
$$


Concerning initial conditions, as model case one usually considers well-prepared data, i.e. in the form:

$$
u_{0}^{\varepsilon}(x):=q^{\varepsilon}\left(\tilde{d}\left(x, \Sigma_{0}\right)\right) \quad(x \in M),
$$

where $\tilde{d}\left(\cdot, \Sigma_{0}\right)$ is the signed distance from the smooth boundary $\Sigma_{0}$ of a bounded domain $E_{0} \subset \mathbb{R}^{N}$, defined by

$\tilde{d}\left(x, \Sigma_{0}\right):=d\left(x, \Sigma_{0}\right) \quad$ if $x \in E_{0}, \quad \tilde{d}\left(x, \Sigma_{0}\right):=-d\left(x, \Sigma_{0}\right) \quad$ if $x \in \mathbb{R}^{N} \backslash E_{0}$,

possibly regularizing the signed distance far away from the initial interface $\Sigma_{0}$.

In [31] it is proved that when $\varepsilon>0$ is sufficiently small and $u^{\varepsilon}$ solves problem (1.3)-(1.4), the energy density

$$
d \mu_{t}^{\varepsilon}:=\left\{\frac{\varepsilon}{2}\left|\nabla u^{\varepsilon}\right|^{2}+\frac{1}{\varepsilon} F\left(u^{\varepsilon}\right)\right\} d x,
$$

satisfies both a Brakke's and a Huisken's type formula, in analogy to Brakke's inequality and Huisken's monotonicity formula for a family $\left\{\Sigma_{t}\right\}_{t \geq 0}$ of hypersurfaces of $\mathbb{R}^{N}$ that evolve by mean curvature flow. However, such formulas for $d \mu_{t}^{\varepsilon}$ involve a new term: the discrepancy Radon measure

$$
d \xi_{t}^{\varepsilon}:=\left\{\frac{\varepsilon}{2}\left|\nabla u^{\varepsilon}\right|^{2}-\frac{1}{\varepsilon} F\left(u^{\varepsilon}\right)\right\} d x .
$$

A crucial point in [31] is to show that

$$
d \xi_{t}^{\varepsilon} \leq 0 \text { for all } t \geq 0, \varepsilon>0 .
$$

This inequality, following [36] in the stationary case, is deduced from the inequality

$$
\left|\nabla r^{\varepsilon}(x, t)\right| \leq 1 \text { for all } x \in \mathbb{R}^{N}, t \geq 0,
$$

where $r^{\varepsilon}: \mathbb{R}^{N} \times(0, \infty)$ is the function defined by

$$
u^{\varepsilon}(x, t)=q^{\varepsilon}\left(r^{\varepsilon}(x, t)\right) \text { for all } x \in \mathbb{R}^{N}, t>0 .
$$

Note that, by hypothesis (1.7) on initial conditions $u_{0}^{\varepsilon}$, inequality (1.9) is satisfied for all $x \in \mathbb{R}^{N}$ and $t=0$, since $x \mapsto \tilde{d}\left(x, \Sigma_{0}\right)$ is 1-Lipschitz. Then by maximum principle, applied to a certain parabolic equation satisfied by $z:=\left|\nabla r^{\varepsilon}\right|^{2}$, it is obtained for all $x \in \mathbb{R}^{N}, t>0$.

As a consequence of Huisken's type monotonicity formula and (1.8), there holds

$$
\frac{d}{d t} \int_{\mathbb{R}^{N}} \psi(x, t) d \mu_{t}^{\varepsilon}(x) \leq 0,
$$


i.e., monotonicity of the function $t \mapsto \int_{\mathbb{R}^{N}} \psi(x, t) d \mu_{t}^{\varepsilon}(x)$; here, for each fixed $y \in \mathbb{R}^{N}, s>0$,

$$
\psi(x, t) \equiv \psi(x, t ; y, s):=\frac{e^{-\frac{|x-y|^{2}}{4(s-t)}}}{[4 \pi(s-t)]^{\frac{N-1}{2}}} \quad \text { for all } x \in \mathbb{R}^{N}, 0 \leq t<s ;
$$

observe that this function $\psi$ is, up to a multiplicative factor $\sqrt{4 \pi(s-t)}$, exactly the backward heat kernel in dimension $N$.

Next it is shown that then there are a Radon measure $\mu_{t}$ on $\mathbb{R}^{N}$ and a sequence $\left\{\varepsilon_{n}\right\} \subset(0, \infty), \varepsilon_{n} \rightarrow 0$ as $n \rightarrow \infty$ such that, for every $t>0, \mu_{t}^{\varepsilon_{n}}$ converges as Radon measure on $\mathbb{R}^{N}$ to $\mu_{t}$ for all $t \geq 0$ as $n \rightarrow \infty$. These measures are shown to be $(N-1)$-rectifiable, as a consequence of density bounds derived from (1.10). Finally, Brakke's inequality for $d \mu_{t}$ is obtained from the corresponding approximate ones valid for $d \mu_{t}^{\varepsilon}$.

The aim of this paper and of [41] is to generalize the results in [31] recalled above, to the case of solutions $u^{\varepsilon}$ to problem (1.1)-(1.2) on Riemannian manifolds. We always assume that there exists $\lambda \in \mathbb{R}$ such that

$$
\operatorname{Ric}(X, X) \geq \lambda\langle X, X\rangle \text { for all } X \in T_{x} M, x \in M \text {; }
$$

here Ric denotes the Ricci tensor on $M$. Note that for $M=\mathbb{R}^{N}$, we have $\lambda=0$; for the hyperbolic space $\mathbb{H}^{N}, \lambda=-(N-1)$; for the sphere $\mathbb{S}^{N}, \lambda=N-1$ (see Subsection 2). Indeed, in these cases (1.12) holds with the equality sign.

Observe that, under the assumption on the Ricci curvature, we can apply comparison principle for (1.1)-(1.2), obtaining uniqueness of solutions. In addition, we can treat not only well-prepared initial conditions, but also quite general initial conditions. Hence our results with $M=\mathbb{R}^{N}$ extend those in [31] in this respect.

Note that while mean curvature flow has been investigated also on Riemannian manifolds (see, e.g. $[2,27,28,30]$ ), to the best of our knowledge, the question of approximation of mean curvature flow via Allen-Cahn equation on Riemannian manifolds has not been addressed. On the other hand, the connection between the stationary Allen-Cahn equation and minimal hypersurfaces has been widely studied e.g. in $[16,39]$ and [40].

Now, we outline results that will be shown in the present paper and we briefly mention the content of [41]. For any $\varepsilon>0$, define the energy density

$$
d \mu_{t}^{\varepsilon}:=\left\{\frac{\varepsilon}{2}\left|\nabla u^{\varepsilon}\right|^{2}+\frac{1}{\varepsilon} F\left(u^{\varepsilon}\right)\right\} d \mathcal{V}(x),
$$

$u^{\varepsilon}$ being a solution to equation (1.1) and $d \mathcal{V}$ the volume element on $M$. For $d \mu_{t}^{\varepsilon}$ we shall prove Brakke's and Huisken's type formulas (see Lemma 5.2, and respectively, Lemma 5.7). Also in this case, they contain the discrepancy Radon measure

$$
d \xi_{t}^{\varepsilon}:=\left(\frac{\varepsilon}{2}\left|\nabla u^{\varepsilon}\right|^{2}-\frac{1}{\varepsilon} F\left(u^{\varepsilon}\right)\right) d \mathcal{V}(x) .
$$


For a general class of initial conditions, without supposing that $u_{0}^{\varepsilon}$ are somehow well-prepared, we prove that

$$
\limsup _{\varepsilon \rightarrow 0^{+}} \sup _{(x, t) \in Q} \xi_{t}^{\varepsilon}(x) \leq 0,
$$

for each compact subset $Q \subset M \times(0, \infty)$. To do this, we slightly improve an elementary but very clever idea from [35] and [29]. Observe that in [29] the stationary problem in bounded domains of $\mathbb{R}^{N}$ is addressed. Moreover, suitably localizing the argument from [35], it is shown that the positive part of $\xi^{\varepsilon}$ is bounded, uniformly with respect to $\varepsilon$.

However, for well-prepared initial conditions $u_{0}^{\varepsilon}$ also an alternative strategy can be used. In fact, in Section 4 we prove for properly well-prepared initial conditions an asymptotic control of discrepancy, by methods similar to those used in [31]. However, some differences from [31] occur, for the presence of the general Riemannian metric on $M$, which we describe below.

As a consequence, instead of $q_{\varepsilon}$, it is convenient to consider the one-dimensional profile $h^{\varepsilon}$, which is the solution, for any $\varepsilon>0$, to problem

$$
\left\{\begin{array}{l}
\frac{1}{\varphi}\left\{\varphi h_{\varepsilon}^{\prime}\right\}^{\prime}=f_{\varepsilon}\left(h_{\varepsilon}\right) \quad \text { in }(0,1) \\
h_{\varepsilon}(0)=0, \quad h_{\varepsilon}(1)=1,
\end{array}\right.
$$

(see Subsection 4.1) where $\varphi:[0,1] \rightarrow(0, \infty)$ is an increasing convex smooth function such that $\varphi(0) \geq 1, \varphi^{\prime}(0)=0$ that will be chosen to balance some curvature effects. We still denote by $h_{\varepsilon}$ the odd reflection of the solution of (1.15). Indeed, note that the ordinary differential equation in (1.15), for the choice $\varphi \equiv 1$, coincides with that solved by $q_{\varepsilon}$ but for technical reasons it is more convenient to consider (1.15) on a bounded interval. As a preliminary step we shall prove that (see Subsection 4.1)

$$
\limsup _{\varepsilon \rightarrow 0} \sup _{\tau \in(0,1)} \varepsilon\left\{\frac{1}{2} h_{\varepsilon}^{\prime}(\tau)^{2}-F_{\varepsilon}\left(h_{\varepsilon}(\tau)\right)\right\} \leq 0,
$$

exploiting the fact that (1.15) is now solved in a bounded interval.

Concerning the initial conditions $u_{0}^{\varepsilon}$, well-prepared data will be now of the form (see Subsection 4.2):

$$
u_{0}^{\varepsilon}(x):=h^{\varepsilon}\left(\Psi\left(\tilde{d}\left(x, \Sigma_{0}\right)\right)\right) \quad(x \in M),
$$

where $E_{0} \subset M$ is an open bounded subset with smooth boundary $\Sigma_{0}:=\partial E_{0}$. Now, $d\left(x, \Sigma_{0}\right)$ is the Riemannian distance of $x \in M$ to $\Sigma_{0}$, while the signed distance $\tilde{d}\left(x, \Sigma_{0}\right)$ is defined accordingly and $\Psi(\cdot)$ is a suitable smoothed and 1-Lipschitz truncation of the identity, which makes $u_{0}^{\varepsilon}$ constant far from $\Sigma_{0}$, where the distance function is possibly singular. 
Define the function $z^{\varepsilon}: M \times[0, \infty) \rightarrow \mathbb{R}$ by

$$
u^{\varepsilon}(x, t):=h_{\varepsilon}\left(z^{\varepsilon}(x, t)\right) \quad(x \in M, t \geq 0) .
$$

Under the assumption

$$
\left|\nabla z^{\varepsilon}(x, 0)\right| \leq 1 \text { for all } x \in M,
$$

which clearly follows if (1.17) holds, and

$$
\left(\frac{\varphi^{\prime}}{\varphi}\right)^{\prime} \geq 2 \max \{-\lambda, 0\} \quad \text { in }(0,1),
$$

we shall prove that (see Subsection 4.3)

$$
\left|\nabla z^{\varepsilon}(x, t)\right| \leq 1 \quad \text { for all } x \in M, t \geq 0 .
$$

Indeed, note that, in view of (1.19) and (1.20), we can infer that $\tilde{w} \equiv 1$ is a supersolution to a certain parabolic equation solved by $\left|\nabla z^{\varepsilon}\right|^{2}$. Hence, from maximum principle we can prove that (1.21) holds true. This argument fails, if $\varphi \equiv 1$ and $\lambda<0$, because of the presence of an extra term related to the Ricci curvature; so, we cannot consider $q^{\varepsilon}$ instead of $h^{\varepsilon}, e . g$. when $M=\mathbb{H}^{N}$, and this is the reason why we have to introduce the profile $h_{\varepsilon}$ defined in (1.15). As a consequence of (1.16)-(1.21) we have (1.14) for any $Q \subset M \times[0, \infty)$.

Note that both for general initial conditions and for well prepared initial conditions we cannot prove that the discrepancy term is nonpositive, as occurred in (1.8) in the Euclidean space. However, condition (1.14) will play the same role as (1.8) has in the case $M=\mathbb{R}^{N}$.

Then, in Section 5 from Huisken's type equality for the density energy and (1.14) we obtain the following inequality (see Theorem 5.8)

$$
\frac{d}{d t} \int_{M} \phi(x, t) d \mu_{t}^{\varepsilon} \leq \frac{C_{3}}{\sqrt{s-t}} \int_{M} \phi(x, t) d \mu_{t}^{\varepsilon}+C_{4}+\frac{C_{5}}{\sqrt{s-t}}
$$

for all $0<t_{0} \leq t<s$, for some positive constants $C_{3}, C_{4}, C_{5}=C_{5}\left(T_{0}\right)$ independent of $\varepsilon$. Inequality (1.22) is a natural counterpart on a manifold of the monotonicity formula (1.10) but, due to the presence of extra terms, (1.22) does not imply monotonicity for the function $t \rightarrow \int_{M} \phi(x, t) d \mu_{t}^{\varepsilon}(x)$.

Here, for any fixed reference point $(y, s) \in M \times(0, \infty), \phi(x, t) \equiv \phi(x, t ; y, s)$ is a suitable kernel, which replaces (1.11). It depends explicitely on the Riemannian distance $d(x)=d(x, y)$ for $x, y \in M$ as follows

$$
\phi(x, t)=\hat{\zeta}\left(d^{2}(x)\right)(s-t)^{-\frac{N-1}{2}} e^{-\frac{d^{2}(x)}{4(s-t)}},
$$

furthermore, in constrast with the case of $\mathbb{R}^{N}$, it has a suitably small compact support in space due to the cut-off function $\hat{\zeta}$. As a consequence, it allows us to control 
the behavior of $d \mu_{t}^{\varepsilon}$ only at small scales. For this reasons, we shall refer to (1.22) as a local almost monotonicity formula. This choice of the kernel is very natural, since, up to the cut-off and the factor $\sqrt{s-t}$, is nothing but the leading order term in the expansion of the backward heat kernel on the manifold $M$ with pole at $(y, s)$ for short times. It would be very interesting to find a more precise localized monotonicity formula for the Allen-Cahn equation on a manifold containing no error term. It should be analouge to the one in [31] for $\mathbb{R}^{N}$ but local as the celebrated formula in [18] for the mean curvarure flow, still in $\mathbb{R}^{N}$.

As a consequence of (1.22) we obtain, for all $0 \leq t_{0}<t<s$,

$$
\mathcal{G}(t) \leq e^{\frac{C_{3}}{2}\left(\sqrt{s-t_{0}}-\sqrt{s-t}\right)}\left[\mathcal{G}\left(t_{0}\right)+C_{4}\left(t-t_{0}\right)+C_{5}\left(\sqrt{s-t_{0}}-\sqrt{s-t}\right)\right],
$$

where

$$
\mathcal{G}(t):=\int_{M} \phi(x, t) d \mu_{t}^{\varepsilon} \quad(0 \leq t<s)
$$

and this is precisely the inequality needed to have uniform density bounds for the measures $\mu_{t}^{\varepsilon}$ at small scales. We conclude Section 5 giving some useful compactness properties for the solutions $u^{\varepsilon}$ both in $L_{\text {loc }}^{1}$ and in the space of functions of bounded variation.

Finally, let us mention that, out of its independent interest, inequality (1.24) will be used in [41] to prove that there exist a Radon measure $\mu_{t}$ on $M$ and a sequence $\left\{\varepsilon_{n}\right\} \subset(0, \infty), \varepsilon_{n} \rightarrow 0$ as $n \rightarrow \infty$ such that, for every $t>0, \mu_{t}^{\varepsilon_{n}}$ converges as Radon measure on $M$ to $\mu_{t}$ for all $t \geq 0$ as $n \rightarrow \infty$. Moreover, $\mu_{t}$ will be $(N-1)$-rectifiable and they will satisfy the Brakke's inequality, i.e. they will be a generalized solution of the mean curvature flow in the sense of varifolds with the surface measure on $\Sigma_{0}$ as initial data.

\section{Preliminaries from differential geometry}

In this section we recall some basic facts and notations from Riemannian Geometry, that will be used in the sequel and in [41], too (for more details see, e.g., [26,34]).

Let $M$ be an $N$-dimensional Riemannian manifold, equipped with a metric tensor $g$. For any given point $x \in M$, let $T_{x} M$ be the tangent space at $x, T M$ be the tangent bundle, $T_{x}^{*} M$ be the cotangent space at $x, T^{*} M$ be the cotangent bundle, $\Gamma(T M)$ denote the vector space of smooth sections of $T M$, i.e. the smooth vector fields on $M$. In local coordinates $\left\{x^{1}, \ldots, x^{N}\right\}$, we have a natural local basis $\left\{\frac{\partial}{\partial x^{1}}, \ldots, \frac{\partial}{\partial x^{N}}\right\}$ for $T M$. The metric tensor $g=g_{i j} d x^{i} \otimes d x^{j}$ is represented by a smooth matix-valued function $g_{i j}=g\left(\frac{\partial}{\partial x^{i}}, \frac{\partial}{\partial x^{j}}\right)$, so that locally the inner Riemannian product $\langle\cdot, \cdot\rangle$ is given by

$$
\langle X, Y\rangle:=g_{i j} X^{i} Y^{j},
$$

where the vectors $X=X^{i} \frac{\partial}{\partial x^{i}}, Y=Y^{i} \frac{\partial}{\partial x^{i}}$ belong to the tangent space $T_{x} M$ (see [7]). The induced geodesic distance between any two points $x, y \in M$ will be 
indicated by $d(x, y)$. For any $x_{0} \in M, r>0$ let $B_{r}\left(x_{0}\right):=\left\{x \in M \mid d\left(x, x_{0}\right)<\right.$ $r\}$. The gradient $\nabla u$ of a function $u \in C^{1}(M)$ is given by $(\nabla u)^{i}:=g^{i j} \frac{\partial u}{\partial x^{j}}(i=$ $1, \ldots, N)$, so that $d u(X)=\langle X, \nabla u\rangle$ for any $X \in \Gamma(T M)$.

Recall that for any vector field $Y \in \Gamma(T M)$ there exists a unique smooth function on $M$, denoted by $\operatorname{div} Y$, such that the following identity holds:

$$
\int_{M} \phi \operatorname{div} Y d \mathcal{V}=-\int_{M}\langle Y, \nabla \phi\rangle d \mathcal{V}
$$

for all $\phi \in C_{c}^{1}(M)$. Furthermore, in local coordinates $\operatorname{div} Y=\frac{1}{\sqrt{\bar{g}}} \frac{\partial}{\partial x^{k}}\left(\sqrt{\bar{g}} Y^{k}\right)$, where $\bar{g}:=\operatorname{det}\left(g_{i j}\right)$.

The Laplace-Beltrami operator on $M$ is given by

$$
\Delta=\operatorname{div} \circ \nabla=\frac{1}{\sqrt{\bar{g}}} \frac{\partial}{\partial x^{i}}\left(\sqrt{\bar{g}} g^{i j} \frac{\partial}{\partial x^{j}}\right) .
$$

The Levi-Civita connection D of the metric $g$ is given by $\mathrm{D}_{\frac{\partial}{\partial x^{i}}} \frac{\partial}{\partial x^{j}}=\Gamma_{i j}^{k} \frac{\partial}{\partial x^{k}}$, where

$$
\Gamma_{i j}^{k}:=\frac{1}{2} g^{k l}\left(\frac{\partial g_{j l}}{\partial x^{i}}+\frac{\partial g_{i l}}{\partial x^{j}}-\frac{\partial g_{i j}}{\partial x^{l}}\right)
$$

are the Christoffel symbols.

We also recall that the Hessian of $f \in C^{2}(M ; \mathbb{R})$ is the symmetric endomorphism of $T M$ defined by

$$
\text { Hess } f(X):=\mathrm{D}_{X} \nabla f \quad \text { for any } X \in \Gamma(T M),
$$

or its associated symmetric bilinear form on $T M$ defined by

$$
(\text { Hess } f)(X, Y):=X(Y(f))-\mathrm{D}_{X} Y(f) \text { for any } X, Y \in \Gamma(T M) \text {. }
$$

We have:

$$
(\text { Hess } f)(X, Y)=\left\langle\mathrm{D}_{X}(\nabla f), Y\right\rangle \text { for any } X, Y \in \Gamma(T M) .
$$

Also, in local coordinates, there holds

$$
(\text { Hess } f)(X, Y)=\sum_{i, j=1, N} X^{i} X^{j}(\operatorname{Hess} f)_{i j},
$$

where

$$
(\operatorname{Hess} f)_{i j}=(\operatorname{Hess} f)\left(\frac{\partial}{\partial x^{i}}, \frac{\partial}{\partial x^{j}}\right)=\frac{\partial^{2} f}{\partial x^{i} \partial x^{j}}-\Gamma_{i j}^{k} \frac{\partial f}{\partial x^{k}} \text {. }
$$


In terms of the Hessian, the Laplace-Beltrami operator rewrites as

$$
\Delta f=\sum_{i, j=1}^{N} g^{i j}(\operatorname{Hess} f)\left(\frac{\partial}{\partial x^{i}}, \frac{\partial}{\partial x^{j}}\right)=\operatorname{tr}(\operatorname{Hess} f) ;
$$

here and hereafter tr denotes the trace operator (taken fiberwise).

For any $y \in M$, denote by $\operatorname{inj}(y)$ the injectivity radius at $y$. In the sequel we use the next lemma.

Lemma 2.1. Let $y \in M, d(x):=d(x, y)$ for all $x \in M$. For any compact subset $K \subset M$ there exists a constant $C>0$ such that if

$$
y \in K, \quad d(x) \leq \frac{1}{2} \inf _{y \in K} \operatorname{inj}(y),
$$

then

$$
\left\|\frac{1}{2} \operatorname{Hess} d^{2}(x)(X, X)-g(X, X)\right\| \leq C d^{2}(x)\|X\|^{2} \quad \text { for any } X \in T_{x} M,
$$

and

$$
\left|\frac{1}{2} \Delta d^{2}(x)-N\right| \leq C d^{2}(x)
$$

For $u, v, \phi \in C^{2}(M ; \mathbb{R})$, it is direct to see that:

$$
\langle\nabla u, \nabla\langle\nabla \phi, \nabla v\rangle)=(\operatorname{Hess} \phi)(\nabla u, \nabla v)+(\operatorname{Hess} v)(\nabla u, \nabla \phi) .
$$

The curvature tensor of the Levi-Civita connection $\mathrm{D}$ is given by

$$
R(X, Y) Z:=\mathrm{D}_{X} \mathrm{D}_{Y} Z-\mathrm{D}_{Y} \mathrm{D}_{X} Z-\mathrm{D}_{[X, Y]} Z \text { for any } X, Y, Z \in \Gamma(T M) ;
$$

in local coordinates, $R\left(\frac{\partial}{\partial x^{i}}, \frac{\partial}{\partial x^{j}}\right) \frac{\partial}{\partial x^{l}}=R_{l i j}^{k} \frac{\partial}{\partial x^{k}}$, where

$$
R_{l i j}^{k}:=\frac{\partial \Gamma_{j l}^{k}}{\partial x^{i}}-\frac{\partial \Gamma_{i l}^{k}}{\partial x^{j}}+\Gamma_{i m}^{k} \Gamma_{j l}^{m}-\Gamma_{j m}^{k} \Gamma_{i l}^{m} .
$$

The sectional curvature of the plane $X \wedge Y$ spanned by the linearly independent tangent vectors $X=X^{i} \frac{\partial}{\partial x^{i}}, Y=Y^{i} \frac{\partial}{\partial x^{i}} \in T_{x} M$ is

$$
K(X \wedge Y):=\frac{\langle R(X, Y) X, Y\rangle}{|X \wedge Y|^{2}},
$$

where $|X \wedge Y|^{2}=|X|^{2}|Y|^{2}-\langle X, Y\rangle^{2}$. The Ricci curvature in the direction $X=$ $X^{i} \frac{\partial}{\partial x^{i}} \in T_{x} M$ is

$$
\operatorname{Ric}(X, X):=g^{j l}\left\langle R\left(X, \frac{\partial}{\partial x^{j}}\right) X, \frac{\partial}{\partial x^{l}}\right\rangle
$$


the Ricci tensor is

$$
R_{i k}=g^{j l} R_{i j k l}=R_{k i}
$$

where $R_{i j k l}=g_{i m} R_{j k l}^{m}$.

Furthermore, recall the Bochner-Weitzenböch formula : for $\phi \in C^{2}(M ; \mathbb{R})$ there holds

$$
\frac{1}{2} \Delta\left(|\nabla \phi|^{2}\right)=|\operatorname{Hess} \phi|^{2}+\langle\nabla \phi, \nabla \Delta \phi\rangle+\operatorname{Ric}(\nabla \phi, \nabla \phi) .
$$

\section{Asymptotic control of discrepancy for general initial conditions}

\subsection{General initial conditions}

Let $E_{0} \subset M$ be an open bounded subset with $C^{2}$-boundary $\partial E_{0}=\Sigma_{0}$. Note that there exist $R_{0}>0, C_{0}>0$ such that

$$
\mathcal{H}^{N-1}\left(\Sigma_{0} \cap B_{R}(x)\right) \leq C_{0} \omega_{N-1} R^{N-1}
$$

for all $0<R<R_{0}$.

For any $\varepsilon>0$ set

$$
E^{\varepsilon}(x, t):=\frac{1}{2}\left|\nabla u^{\varepsilon}\right|^{2}+\frac{1}{\varepsilon^{2}} F\left(u^{\varepsilon}\right) \quad(x \in M, t \geq 0)
$$

clearly (see (1.13)),

$$
d \mu_{t}^{\varepsilon}(x)=\varepsilon E^{\varepsilon}(x, t) d \mathcal{V}(x) \quad(x \in M, t \geq 0) .
$$

Concerning the initial conditions $u_{0}^{\varepsilon}$ (and the corresponding $\mu_{0}^{\varepsilon} \equiv \mu^{\varepsilon}(\cdot, 0)$ given by (1.13)) we always assume the following:

$\left(H_{1}\right)\left\{\begin{array}{c}\text { for all } x \in M, 0<R<R_{0}, 0<\varepsilon<1 ; \\ \text { (iv) there exists } k_{0}>0 \text { such that }\left\|u_{0}^{\epsilon}\right\|_{\infty} \leq k_{0}\end{array}\right.$

(i) $\mu_{0}^{\varepsilon} \rightarrow \alpha \mathcal{H}^{N-1}\left\lfloor\Sigma_{0}\right.$ as $\varepsilon \rightarrow 0$ as Radon measures, for some $\alpha \geq 0$;

(ii) $u_{0}^{\varepsilon} \rightarrow 2 \chi_{E_{0}}-1$ as $\varepsilon \rightarrow \infty$ in $B V_{\text {loc }}(M)$ weakly $-*$;

(iii) there exists $C_{0}>0$ such that $\frac{\mu_{0}^{\varepsilon}\left(B_{R}(x)\right)}{\omega_{N-1} R^{N-1}} \leq C_{0}$

(v) $u_{0}^{\varepsilon} \in C^{1}(M)$ and there exists $\check{C}>0$ such that for any $0<\varepsilon<1\left\|\nabla u_{0}^{\varepsilon}\right\|_{\infty} \leq \frac{\check{C}}{\varepsilon}$.

Throughout this section, we will not assume any further structure assumption on the initial data and, on the contrary, even the previous regularity assumptions both on $\Sigma_{0}$ and on $u_{0}^{\varepsilon}$ could be further relaxed. 


\subsection{Global existence and uniqueness results}

Concerning existence and uniqueness of solutions to problem (1.1)-(1.2) we state the next Proposition.

Proposition 3.1. Let the hypotheses $\left(H_{0}\right),\left(H_{1}\right)$ be satisfied. Then problem (1.1)(1.2) admits a unique bounded solution. Moreover, $u^{\varepsilon} \in C^{\infty}(M \times(0, \infty)) \cap$ $C^{0}(M \times[0, \infty))$, and

$$
\left|u^{\varepsilon}\right| \leq k_{0} \quad \text { for all } x \in M, t>0 .
$$

In addition,

$$
\sup _{\varepsilon>0} \sup _{t \in(0, \infty)} \varepsilon \int_{M} E^{\varepsilon} d \mathcal{V} \leq C_{2}
$$

where $C_{2}:=\sup _{\varepsilon>0} \mu_{0}^{\varepsilon}(M)$;

$$
t \mapsto \int_{M} E^{\varepsilon}(x, t) d \mathcal{V}(x) \text { is nonincreasing for } t>0 .
$$

Proof. Existence and regularity of solutions can be shown by usual methods, e.g. solving the corresponding IBV problems on an increasing family of bounded domains with smooth boundary and arguing by local a-priori estimates and compactness. In view of (1.12), from results in [17] uniqueness and comparison principles for problem (1.1)-(1.2) can be easily deduced. In view of $\left(H_{0}\right)-(i i)$, the functions $\bar{v} \equiv k_{0}, \underline{v} \equiv-k_{0}$ are a supersolution and, respectively, a subsolution to problem (1.1), (1.2). Hence, by comparison principle (3.3) follows. Finally, inequality (3.4) and the property (3.5) follows passing to the limit in the global energy inequality on the approximating domains.

Proposition 3.2. Let the hypotheses $\left(H_{0}\right),\left(H_{1}\right)-(i v)$ be satisfied. Let $u^{\varepsilon}$ be the solution to problem (1.1)-(1.2). Then (3.3) holds true. Furthermore, for any compact subset $K \subset M$ and for any $\tau \in(0, T)$ there exists a constant $\tilde{k}>0$ such that

$$
\begin{gathered}
\left\|\nabla u^{\varepsilon}(\cdot, t)\right\|_{L^{\infty}(K)} \leq \frac{\tilde{k}}{\varepsilon} \quad \text { for all } t \in(\tau, T) ; \\
\varepsilon \xi^{\varepsilon}(x, t) \leq \tilde{k} \quad \text { for all } x \in K, t \in(\tau, T) .
\end{gathered}
$$

Proof. Note that (3.3) can be deduced as in the proof of Proposition 3.1. Moreover, (3.6) follows by standard parabolic estimates, writing the equation in local coordinates and arguing by scaling. Consequently,(3.7) is obtained, in view of (3.3). The proof is complete. 
Remark 3.3. For further references, note that from $\left(H_{1}\right)-(i)$ and (3.5) it is direct to see that, for each compact subset $K \subset M, T>0, \tau \in[0, T)$, there holds:

$$
\sup _{\varepsilon>0} \sup _{t \in(\tau, T)} \varepsilon \int_{K} E^{\varepsilon} d \mathcal{V} \leq \bar{C}
$$

for some constant $\bar{C}>0$ depending on the compact subset $K, \tau>0, T>0$, and independent of $\varepsilon>0$. Indeed, under $\left(H_{1}\right)-(i)$ we have

$$
\bar{C} \leq C_{2},
$$

where $C_{2}$ given in Proposition 3.1 is clearly independent of $K, \tau>0, T>0$ in view of (3.4) and (3.5). However, in the sequel most of the time the arguments will rely only on (3.8) and we shall not use the property (3.9).

\subsection{Asymptotic control of discrepancy}

We prove the next result.

Proposition 3.4. Let assumption $\left(H_{0}\right)$ hold true. Let $\left\{u^{\varepsilon}\right\}$ be a family of uniformly bounded solutions to problem (1.1)-(1.2), i.e. (3.3) is verified. Then (1.14) is satisfied.

In order to prove Proposition 3.4 we need some preliminary results.

Lemma 3.5. Let $\bar{x} \in M, r>0, \bar{t}>4 r^{2}$. Let $\Omega_{0}=B_{4 r}(\bar{x}) \times\left(\bar{t}-4 r^{2}, \bar{t}\right], \Omega:=$ $B_{r}(\bar{x}) \times\left(\bar{t}-r^{2}, \bar{t}\right]$. Assume that, for some $C>0$,

$$
\sup _{0<\varepsilon<1}\left\|u^{\varepsilon}\right\|_{L^{\infty}\left(\Omega_{0}\right)} \leq C .
$$

Then, for any $\sigma_{0} \in(0,2)$, there exists a constant $C_{0}=C_{0}\left(\Omega, C, \sigma_{0}\right)>0$ such that

$$
\left\|u^{\varepsilon}\right\|_{L^{\infty}(\Omega)} \leq 1+C_{0} \varepsilon^{\sigma_{0}} \quad \text { for any } \varepsilon \in(0,1) .
$$

Proof. Fix any $\sigma_{0} \in(0,2)$. It suffices to show the thesis with $C_{0}=1$ and $\varepsilon \rightarrow 0^{+}$. In fact, as a consequence of this, we can immediately get (3.11), taking possibly a bigger $C_{0}$.

Suppose, by contradiction, that there exists a sequence $\left\{\varepsilon_{n}\right\} \subset(0,1)$ such that $\varepsilon_{n} \rightarrow 0^{+}$as $n \rightarrow \infty$ and $\sup _{\Omega} u^{\varepsilon_{n}} \geq 1+\varepsilon_{n}^{\sigma_{0}}$. The case $\inf _{\Omega} u^{\varepsilon_{n}} \geq-1-\varepsilon_{n}^{\sigma_{0}}$ can be treated with obvious modifications; so we do not discuss it in details.

Let $\Omega_{1}:=B_{2 r}(\bar{x}) \times\left(\bar{t}-2 r^{2}, \bar{t}\right]$. For any $n \in \mathbb{N}$ select $\varphi_{n} \in C^{\infty}\left(\bar{\Omega}_{0}\right)$ such that

$$
\begin{array}{rlrl}
\varphi_{n} & \equiv 1+\frac{1}{2} \varepsilon_{n}^{\sigma_{0}} & & \text { in } \Omega, \\
1<1+\frac{1}{2} \varepsilon_{n}^{\sigma_{0}} \leq \varphi_{n} \leq 1+C & & \text { in } \Omega_{0}, \\
\varphi_{n} & \equiv 1+C & & \text { in } \Omega_{0} \backslash \Omega_{1} ;
\end{array}
$$


moreover, for some $\bar{C}>0$, for all $n \in \mathbb{N}$,

$$
\left|\nabla \varphi_{n}\right|+\left|\operatorname{Hess}\left(\varphi_{n}\right)\right|+\left|\partial_{t} \varphi_{n}\right| \leq \bar{C} \quad \text { in } \Omega_{0}
$$

Set $g_{n}:=u^{\varepsilon_{n}}-\varphi_{n}$, so that

$$
g_{n} \leq-1 \text { in }\left[B_{4 r}(\bar{x}) \times\left\{\bar{t}-4 r^{2}\right\}\right] \cup\left[\partial B_{4 r}(\bar{x}) \times\left(\bar{t}-4 r^{2}, \bar{t}\right]\right]
$$

Furthermore,

$$
\sup _{\Omega_{0}} g_{n} \geq \sup _{\Omega} g_{n} \geq \frac{1}{2} \varepsilon_{n}^{\sigma_{0}}>0 .
$$

Then $\max _{\bar{\Omega}_{0}} g_{n}=g_{n}\left(x_{n}, t_{n}\right)$ for some $\left(x_{n}, t_{n}\right) \in \Omega_{0}$. Thus, using (1.1), the fact that $u^{\varepsilon_{n}}\left(x_{n}, t_{n}\right)>1,\left(H_{0}\right)-(i v)$ and (3.12) we obtain

$$
\begin{aligned}
0 \geq & \Delta g\left(x_{n}, t_{n}\right)-\partial_{t} g\left(x_{n}, t_{n}\right)=\Delta u^{\varepsilon_{n}}\left(x_{n}, t_{n}\right)-\partial_{t} u^{\varepsilon_{n}}\left(x_{n}, t_{n}\right) \\
& +\partial_{t} \varphi_{n}\left(x_{n}, t_{n}\right)-\Delta \varphi_{n}\left(x_{n}, t_{n}\right) \\
= & \frac{F^{\prime}\left(u^{\varepsilon_{n}}\left(x_{n}, t_{n}\right)\right)}{\varepsilon_{n}^{2}}+\partial_{t} \varphi_{n}\left(x_{n}, t_{n}\right)-\Delta \varphi_{n}\left(x_{n}, t_{n}\right) \\
\geq & \frac{F^{\prime}\left(u^{\varepsilon_{n}}\left(x_{n}, t_{n}\right)\right)}{\varepsilon_{n}^{2}}-\frac{F^{\prime}\left(\varphi_{n}\left(x_{n}, t_{n}\right)\right)}{\varepsilon_{n}^{2}}+\partial_{t} \varphi_{n}\left(x_{n}, t_{n}\right)-\Delta \varphi_{n}\left(x_{n}, t_{n}\right) \\
\geq & \left.\frac{F^{\prime}\left((1-s) \varphi_{n}+s u^{\varepsilon_{n}}\right)}{\varepsilon_{n}^{2}}\left(x_{n}, t_{n}\right)\right|_{s=0} ^{s=1}+\partial_{t} \varphi_{n}\left(x_{n}, t_{n}\right)-\Delta \varphi_{n}\left(x_{n}, t_{n}\right) \\
= & \int_{0}^{1} \frac{d}{d s} \frac{F^{\prime}\left((1-s) \varphi_{n}+s u^{\varepsilon_{n}}\right)}{\varepsilon_{n}^{2}}\left(x_{n}, t_{n}\right) d s+\partial_{t} \varphi_{n}\left(x_{n}, t_{n}\right)-\Delta \varphi_{n}\left(x_{n}, t_{n}\right) \\
= & \frac{g_{n}\left(x_{n}, t_{n}\right)}{\varepsilon_{n}^{2}} \int_{0}^{1} F^{\prime \prime}\left((1-s) \varphi_{n}+s u^{\varepsilon_{n}}\right)\left(x_{n}, t_{n}\right) d s+\partial_{t} \varphi_{n}\left(x_{n}, t_{n}\right)-\Delta \varphi_{n}\left(x_{n}, t_{n}\right) \\
\geq & \frac{1}{2} \inf _{1<s<1+C} F^{\prime \prime}(s) \varepsilon_{n}^{\sigma_{0}-2}-\bar{C} .
\end{aligned}
$$

This is clearly impossible for $n \in \mathbb{N}$ large enough, hence the thesis follows.

$$
\begin{gathered}
\text { Define } G(u):=\varepsilon^{\sigma}\left(2 H_{0}-u^{2}\right) \text {, so that } G>0, G^{\prime \prime}=-2 \varepsilon^{\sigma}<0 \text {. Set } \\
\phi_{G}^{\varepsilon}:=\varepsilon \xi_{t}^{\varepsilon}-G=\frac{\varepsilon^{2}}{2}\left|\nabla u^{\varepsilon}\right|^{2}-F\left(u^{\varepsilon}\right)-G\left(u^{\varepsilon}\right),
\end{gathered}
$$


where $u^{\varepsilon}$ is a solution to equation (1.1). Hence, we have:

$$
\begin{aligned}
\left(\Delta-\partial_{t}\right) \phi_{G}^{\varepsilon}= & \left(\Delta-\partial_{t}\right) \frac{\varepsilon^{2}}{2}\left|\nabla u^{\varepsilon}\right|^{2}-\left(\Delta-\partial_{t}\right)(F+G) \\
= & \varepsilon^{2}\left[\left|\operatorname{Hess} u^{\varepsilon}\left(\nabla u^{\varepsilon}, \nabla u^{\varepsilon}\right)\right|^{2}+\left\langle\nabla u^{\varepsilon}, \nabla \Delta u^{\varepsilon}\right\rangle\right. \\
& \left.+\operatorname{Ric}\left(\nabla u^{\varepsilon}, \nabla u^{\varepsilon}\right)-\left\langle\nabla u^{\varepsilon}, \nabla \partial_{t} u^{\varepsilon}\right\rangle\right]+\left(F^{\prime}+G^{\prime}\right) \partial_{t} u^{\varepsilon} \\
& -\operatorname{div}\left(\left(F^{\prime}+G^{\prime}\right) \nabla u^{\varepsilon}\right) \\
= & \varepsilon^{2}\left[\left|\operatorname{Hess} u^{\varepsilon}\left(\nabla u^{\varepsilon}, \nabla u^{\varepsilon}\right)\right|^{2}+\left\langle\nabla u^{\varepsilon}, \nabla\left(\Delta u^{\varepsilon}-\partial_{t} u^{\varepsilon}\right)\right\rangle\right. \\
& \left.\quad+\operatorname{Ric}\left(\nabla u^{\varepsilon}, \nabla u^{\varepsilon}\right)\right] \\
& +\left(F^{\prime}+G^{\prime}\right)\left(\partial_{t} u^{\varepsilon}-\Delta u^{\varepsilon}\right)-\left(F^{\prime \prime}+G^{\prime \prime}\right)\left|\nabla u^{\varepsilon}\right|^{2} \\
= & \varepsilon^{2}\left|\operatorname{Hess} u^{\varepsilon}\left(\nabla u^{\varepsilon}, \nabla u^{\varepsilon}\right)\right|^{2}+\varepsilon^{2} \operatorname{Ric}\left(\nabla u^{\varepsilon}, \nabla u^{\varepsilon}\right) \\
& -\frac{1}{\varepsilon^{2}}\left(F^{\prime}+G^{\prime}\right) F^{\prime}-G^{\prime \prime}\left|\nabla u^{\varepsilon}\right|^{2} .
\end{aligned}
$$

Note that

$$
\left|\nabla u^{\varepsilon}\right|^{2}\left|\operatorname{Hess} u^{\varepsilon}\left(\nabla u^{\varepsilon}, \nabla u^{\varepsilon}\right)\right|^{2} \geq\left.\left.\frac{1}{4}|\nabla| \nabla u^{\varepsilon}\right|^{2}\right|^{2} .
$$

To see this, take any $p \in M$ and fix an orthonormal frame $\left\{E_{i}\right\}_{i=1, \ldots, N}$ around $p$. Thus,

$$
\begin{gathered}
\left|\nabla u^{\varepsilon}\right|^{2}\left|\operatorname{Hess} u^{\varepsilon}\left(\nabla u^{\varepsilon}, \nabla u^{\varepsilon}\right)\right|^{2}=\sum_{i=1}^{N}\left|\nabla u^{\varepsilon}\right|^{2}\left|\mathrm{D}_{E_{i}} \nabla u^{\varepsilon}\right|^{2} \\
\geq \sum_{i=1}^{N}\left(\left\langle\nabla u^{\varepsilon}, \mathrm{D}_{E_{i}} \nabla u^{\varepsilon}\right\rangle\right)^{2}=\frac{1}{2} \sum_{i=1}^{N}\left(E_{i}\left|\nabla u^{\varepsilon}\right|^{2}\right)^{2}=\left.\left.\frac{1}{4}|\nabla| \nabla u^{\varepsilon}\right|^{2}\right|^{2} .
\end{gathered}
$$

So, (3.14) has been verified. From (3.13), (3.14) and (1.12) we deduce that, whenever $\nabla u^{\varepsilon} \neq 0$,

$$
\begin{gathered}
\varepsilon^{2}\left|\operatorname{Hess} u^{\varepsilon}\left(\nabla u^{\varepsilon}, \nabla u^{\varepsilon}\right)\right|^{2} \geq \frac{1}{\varepsilon^{2}\left|\nabla u^{\varepsilon}\right|^{2}}\left|\nabla\left(\phi_{G}^{\varepsilon}+F+G\right)\right|^{2} \\
\geq 2 \frac{F^{\prime}+G^{\prime}}{\varepsilon^{2}\left|\nabla u^{\varepsilon}\right|} \frac{\nabla u^{\varepsilon}}{\left|\nabla u^{\varepsilon}\right|} \nabla \phi_{G}^{\varepsilon}+\frac{1}{\varepsilon^{2}}\left(F^{\prime}+G^{\prime}\right)^{2},
\end{gathered}
$$

therefore,

$$
\begin{gathered}
\left(\Delta-\partial_{t}\right) \phi_{G}^{\varepsilon}-2 \frac{F^{\prime}+G^{\prime}}{\varepsilon^{2}\left|\nabla u^{\varepsilon}\right|}\left\langle\frac{\nabla u^{\varepsilon}}{\left|\nabla u^{\varepsilon}\right|}, \nabla \phi_{G}^{\varepsilon}\right\rangle \\
\geq \frac{1}{\varepsilon^{2}}\left(G^{\prime}\right)^{2}+\frac{1}{\varepsilon^{2}} F^{\prime} G^{\prime}+\lambda\left|\nabla u^{\varepsilon}\right|^{2} \varepsilon^{2}-G^{\prime \prime}\left|\nabla u^{\varepsilon}\right|^{2} .
\end{gathered}
$$

We summarize these computations in the following: 
Lemma 3.6. Whenever $\nabla u^{\varepsilon} \neq 0$, let

$$
\begin{gathered}
\mathcal{A}^{\varepsilon}:=\left(\Delta-\partial_{t}\right) \phi_{G}^{\varepsilon}+\left(\frac{2 G^{\prime \prime}}{\varepsilon^{2}}-2 \lambda\right) \phi_{G}^{\varepsilon}-\frac{2}{\varepsilon^{2}} \frac{\left(F^{\prime}+G^{\prime}\right)}{\left|\nabla u^{\varepsilon}\right|}\left\langle\frac{\nabla u^{\varepsilon}}{\left|\nabla u^{\varepsilon}\right|}, \nabla \phi_{G}^{\varepsilon}\right\rangle ; \\
\mathcal{B}^{\varepsilon}:=\left(2 \lambda-\frac{2}{\varepsilon^{2}} G^{\prime \prime}\right)(F+G)+\frac{1}{\varepsilon^{2}}\left(G^{\prime}\right)^{2}+\frac{F^{\prime} G^{\prime}}{\varepsilon^{2}} .
\end{gathered}
$$

Then there holds:

$$
\mathcal{A}^{\varepsilon} \geq \mathcal{B}^{\varepsilon} .
$$

The next result is proved by adapting some techniques used in [29].

Lemma 3.7. Let $\bar{x} \in M, r>0, \bar{t}>4 r^{2}$. Let $\Omega_{1}:=B_{2 r}(\bar{x}) \times\left[\bar{t}-2 r^{2}, \bar{t}\right], \Omega:=$ $B_{r}(\bar{x}) \times\left[\bar{t}-r^{2}, \bar{t}\right]$. Suppose that there exist $\gamma \in\left[0, \frac{2}{3}\right)$ and $\bar{C}>0$ such that

$$
\sup _{\Omega_{1}} \varepsilon \xi^{\varepsilon} \leq \bar{C} \varepsilon^{\gamma} \quad \text { for any } 0<\varepsilon<1 .
$$

Then, for any $\sigma \in\left(\gamma, \frac{2}{3}(\gamma+1)\right)$, there exists $C>0$ such that

$$
\sup _{\Omega} \varepsilon \xi^{\varepsilon} \leq C \varepsilon^{\sigma} \quad \text { for any } 0<\varepsilon<1 .
$$

Proof. Fix any $\sigma \in\left(\gamma, \frac{2}{3}(\gamma+1)\right)$. Define $G\left(u^{\varepsilon}\right):=\varepsilon^{\sigma}\left[2 H_{0}-\left(u^{\varepsilon}\right)^{2}\right]$. So, for some $\check{C}>0$, for any $0<\varepsilon<1, \phi_{G}^{\varepsilon} \leq \check{C} \varepsilon^{\gamma}$ in $\Omega_{1}$. We shall prove that, for $\varepsilon \rightarrow 0^{+}$,

$$
\sup _{\Omega} \phi_{G}^{\varepsilon}<\varepsilon^{\sigma} \text {. }
$$

Note that from (3.20) it follows that, for some $C>0$,

$$
\sup _{\Omega} \phi_{G}^{\varepsilon} \leq C \varepsilon^{\sigma} \text { for any } 0<\varepsilon<1,
$$

so, (3.19) follows (possibly taking a bigger $C>0$ ), and in turn this inequality directly yields (3.19), by definition of $\phi_{G}^{\varepsilon}$.

Suppose, by contradiction, that (3.20) is false and that there exists a sequence $\left\{\varepsilon_{n}\right\} \subset(0,1)$ such that $\varepsilon_{n} \rightarrow 0$ as $n \rightarrow \infty$ and $\sup _{\Omega} \phi_{G}^{\varepsilon_{n}} \geq \varepsilon_{n}^{\sigma}$.

Choose $\varphi \in C_{0}^{\infty}\left(\Omega_{1}\right)$ such that $0 \leq \varphi \leq 1, \varphi \equiv 1$ in $\Omega$. Set $\tilde{\phi}^{\varepsilon_{n}}:=\phi_{G}^{\varepsilon_{n}}+$ $\check{C} \varepsilon_{n}^{\gamma} \varphi$. Clearly, in $\left[B_{2 r}(\bar{x}) \times\left\{\bar{t}-2 r^{2}\right\}\right] \cup\left[\partial B_{2 r}(\bar{x}) \times\left(\bar{t}-2 r^{2}, \bar{t}\right]\right]$

$$
\tilde{\phi}^{\varepsilon_{n}} \leq \sup _{\Omega_{1}} \phi_{G}^{\varepsilon_{n}} \leq \check{C} \varepsilon^{\gamma}
$$

Moreover,

$$
\sup _{\Omega_{1}} \tilde{\phi}^{\varepsilon_{n}} \geq \sup _{\Omega} \tilde{\phi}^{\varepsilon_{n}}=\check{C} \varepsilon_{n}^{\gamma}+\sup _{\Omega} \phi_{G}^{\varepsilon_{n}} \geq \check{C} \varepsilon_{n}^{\gamma}+\varepsilon_{n}^{\sigma}>\check{C} \varepsilon_{n}^{\gamma} .
$$


Therefore, $\max _{\bar{\Omega}_{1}} \tilde{\phi}^{\varepsilon_{n}}=\tilde{\phi}^{\varepsilon_{n}}\left(x_{n}, t_{n}\right)$ for some $\left(x_{n}, t_{n}\right) \in \Omega_{1}$. We have:

$$
\tilde{\phi}^{\varepsilon_{n}}\left(x_{n}, t_{n}\right) \geq \check{C} \varepsilon_{n}^{\gamma}+\varepsilon_{n}^{\sigma}>0,
$$

so

$$
\frac{\varepsilon_{n}^{2}}{2}\left|\nabla u^{\varepsilon_{n}}\left(x_{n}, t_{n}\right)\right|^{2} \geq F\left(u^{\varepsilon_{n}}\left(x_{n}, t_{n}\right)\right)+G\left(u^{\varepsilon_{n}}\left(x_{n}, t_{n}\right)\right)+\check{C} \varepsilon_{n}^{\gamma}\left(1-\varphi\left(x_{n}, t_{n}\right)\right)+\varepsilon_{n}^{\sigma} .
$$

Hence, for any $n \in \mathbb{N}$,

$$
\frac{\varepsilon_{n}^{2}}{2}\left|\nabla u^{\varepsilon_{n}}\left(x_{n}, t_{n}\right)\right| \geq \varepsilon_{n}^{1+\frac{\sigma}{2}}>0
$$

Moreover, $\nabla \tilde{\phi}^{\varepsilon_{n}}\left(x_{n}, t_{n}\right)=0$, thus, for some constant $C_{\varphi}>0$, for any $n \in \mathbb{N}$

$$
\left|\nabla \phi_{G}^{\varepsilon_{n}}\left(x_{n}, t_{n}\right)\right| \leq C_{\varphi} \varepsilon_{n}^{\gamma}
$$

We also have

$$
\begin{aligned}
0 \geq & \Delta \tilde{\phi}^{\varepsilon_{n}}\left(x_{n}, t_{n}\right)-\partial_{t} \tilde{\phi}^{\varepsilon_{n}}\left(x_{n}, t_{n}\right)=\check{C} \varepsilon_{n}^{\gamma}\left[\Delta \varphi\left(x_{n}, t_{n}\right)-\partial_{t} \varphi\left(x_{n}, t_{n}\right)\right] \\
& +\Delta \phi_{G}^{\varepsilon_{n}}\left(x_{n}, t_{n}\right)-\partial_{t} \phi_{G}^{\varepsilon_{n}}\left(x_{n}, t_{n}\right)
\end{aligned}
$$

thus, for any $n \in \mathbb{N}$,

$$
\Delta \phi_{G}^{\varepsilon_{n}}\left(x_{n}, t_{n}\right)-\partial_{t} \phi_{G}^{\varepsilon_{n}}\left(x_{n}, t_{n}\right) \leq C_{\varphi} \varepsilon_{n}^{\gamma}
$$

Let $\mathcal{A}^{\varepsilon}, \mathcal{B}^{\varepsilon}$ be defined as in Lemma 3.6. We can find $\bar{\varepsilon}=\bar{\varepsilon}(\lambda)>0$ such that for any $0<\varepsilon<\bar{\varepsilon}$ we have $\frac{G^{\prime \prime}}{\varepsilon^{2}}-2 \lambda<0$. For any $0<\varepsilon<\bar{\varepsilon}$, using (3.21)-(3.23), we also have

$$
\mathcal{A}^{\varepsilon} \leq \tilde{C}\left[\varepsilon_{n}^{\gamma}+\varepsilon_{n}^{\gamma} \varepsilon_{n}^{-1-\frac{\sigma}{2}}\left(\left|F^{\prime}\left(u^{\varepsilon_{n}}\left(x_{n}, t_{n}\right)\right)\right|+\left|G^{\prime}\left(u^{\varepsilon_{n}}\left(x_{n}, t_{n}\right)\right)\right|\right)\right] ;
$$

here and hereafter we always denote by $\tilde{C}$ possibly different constants, independent of $n$ and $\varepsilon$. On the other hand, $\left(2 \lambda-\frac{G^{\prime \prime}}{\varepsilon^{2}}\right)(F+G) \geq 0$. Therefore, if $\frac{1}{2} \leq\left|u^{\varepsilon_{n}}\left(x_{n}, t_{n}\right)\right| \leq 1$, then

$$
\mathcal{B}^{\varepsilon} \geq \tilde{C}\left[\frac{\varepsilon^{2 \sigma}}{\varepsilon^{2}}+\frac{\varepsilon^{\sigma}}{\varepsilon^{2}}\left(\left|F^{\prime}\left(u^{\varepsilon_{n}}\left(x_{n}, t_{n}\right)\right)\right|+\left|G^{\prime}\left(u^{\varepsilon_{n}}\left(x_{n}, t_{n}\right)\right)\right|\right)\right] .
$$

If $\left|u^{\varepsilon_{n}}\left(x_{n}, t_{n}\right)\right| \leq \frac{1}{2}$,

$$
\mathcal{B}^{\varepsilon} \geq \tilde{C} \frac{\varepsilon^{\sigma}}{\varepsilon^{2}}\left(\min _{|s| \leq 1 / 2} F(s)\right)
$$


If $1 \leq\left|u^{\varepsilon_{n}}\left(x_{n}, t_{n}\right)\right| \leq 1+C \varepsilon^{\sigma_{0}}$, then

$$
\mathcal{B}^{\varepsilon} \geq \tilde{C} \frac{\beta_{1} \varepsilon^{2 \sigma}-\beta_{2} \varepsilon^{\sigma+\sigma_{0}}}{\varepsilon^{2}},
$$

for some $\beta_{1}=\beta_{1}\left(H_{0}\right)>0, \beta_{2}=\beta_{2}\left(F^{\prime \prime}\right)>0$, for $\sigma_{0}>\sigma$ fixed.

Clearly, at least one inequality among (3.24), (3.25), (3.26) holds for infinitely many $n \in \mathbb{N}$.

Since $\gamma<\sigma<\frac{2}{3}(\gamma+1)$, we have $\sigma<\gamma+1-\frac{\sigma}{2}$. So, for $n \in \mathbb{N}$ large enough,

$$
\frac{\varepsilon_{n}^{\sigma}}{\varepsilon_{n}^{2}} \geq \tilde{C} \varepsilon_{n}^{\gamma} \varepsilon_{n}^{-1-\frac{\sigma}{2}} .
$$

Therefore, when $\frac{1}{2} \leq\left|u^{\varepsilon_{n}}\left(x_{n}, t_{n}\right)\right| \leq 1$, for $n \in \mathbb{N}$ large enough, we have $\frac{\varepsilon_{n}^{2 \sigma}}{\varepsilon_{n}^{2}} \leq$ $\tilde{C} \varepsilon_{n}^{\gamma}$, so

$$
\varepsilon_{n}^{-2-\gamma+2 \sigma} \leq \tilde{C} .
$$

However, $\gamma<\sigma<\frac{2}{3}(\gamma+1)<2$. Therefore, $-2-\gamma+2 \sigma<-2-\gamma+\frac{4}{3}(\gamma+1)=$ $-\frac{2}{3}+\frac{\gamma}{3}<0$. Hence (3.27) is impossible, for $n \in \mathbb{N}$ large enough.

When $\left|u^{\varepsilon_{n}}\left(x_{n}, t_{n}\right)\right| \leq \frac{1}{2}$,

$$
2 \min _{|s| \leq \frac{1}{2}}\{F(s)\} \frac{\varepsilon^{\sigma}}{\varepsilon^{2}} \leq \mathcal{B}^{\varepsilon} \leq \mathcal{A}^{\varepsilon} \leq \tilde{C}\left(\varepsilon_{n}^{\gamma}+\varepsilon_{n}^{\gamma} \varepsilon_{n}^{-1-\frac{\sigma}{2}}\right) .
$$

Since $\gamma+1-\frac{\sigma}{2}>\sigma$, this yields $\frac{\varepsilon_{n}^{\sigma}}{\varepsilon_{n}^{2}} \leq \tilde{C} \varepsilon_{n}^{\gamma}$, which is again impossible.

When $1 \leq\left|u^{\varepsilon_{n}}\left(x_{n}, t_{n}\right)\right| \leq 1+C \varepsilon^{\sigma_{0}}$, since $\sigma<\sigma_{0}$, from (3.26) we have

$$
\mathcal{B}^{\varepsilon} \geq \tilde{C} \frac{\varepsilon_{n}^{2 \sigma}}{\varepsilon^{2}} .
$$

Furthermore,

$$
\mathcal{A}^{\varepsilon} \leq C\left(\varepsilon_{n}^{\gamma}+\varepsilon_{n}^{\gamma} \varepsilon_{n}^{-1-\frac{\sigma}{2}+\sigma}\right) .
$$

As above it is easily seen that (3.28) and (3.29) are in contrast. This completes the proof.

Proof of Proposition 3.4. Let $\Omega_{0}, \Omega$ be defined as in Lemma 3.7. Moreover, set

$$
\Omega_{2}:=B_{\frac{3}{2} r}(\bar{x}) \times\left[\bar{t}-\frac{3}{2} r^{2}, \bar{t}\right] .
$$

By Proposition 3.2, for some $C_{0}>0$,

$$
\sup _{\Omega_{1}} \varepsilon \xi^{\varepsilon} \leq C_{0} \varepsilon^{0} \quad \text { for all } 0<\varepsilon<1 .
$$


Thus, for any $0<\sigma<\frac{2}{3}$, for some $C_{1}>0$, by Lemma 3.7,

$$
\sup _{\Omega_{2}} \varepsilon \xi^{\varepsilon} \leq C_{1} \varepsilon^{\sigma} \quad \text { for all } 0<\varepsilon<1
$$

Hence, applying once more Lemma 3.7, for any $0<\sigma<\frac{10}{9}$, for some $C>0$,

$$
\sup _{\Omega} \varepsilon \xi^{\varepsilon} \leq C \varepsilon^{\sigma} \quad \text { for all } 0<\varepsilon<1 \text {. }
$$

Now, the conclusion easily follows, choosing $1<\sigma<\frac{10}{9}$ in (3.30).

\section{Asymptotic control of discrepancy for well-prepared initial conditions}

In this section we prove an asymptotic control for the discrepancy $\xi^{\varepsilon}$, using different methods from those in Section 3. To this purpose we need to assume that the initial conditions are properly well prepared (see Subsection 4.2) and the structure of the initial condition will emerge in the next two paragraphs.

\subsection{One-dimensional profile}

Now we study problem $(1.15)$, where $\varphi:[0,1] \rightarrow(0, \infty)$ is an increasing convex smooth function such that $\varphi(0) \geq 1$ and $\varphi^{\prime}(0)=0$. Let us define the energy

$$
\mathcal{E}\left(h_{\varepsilon}\right):=\int_{0}^{1}\left(\frac{1}{2} h_{\varepsilon}^{\prime 2}+F_{\varepsilon}\left(h_{\varepsilon}\right)\right) \varphi(\tau) d \tau .
$$

By the same arguments as in the proof of Proposition 3.1 in [40] it is possible to show next

Lemma 4.1. For any $\varepsilon>0$ there exists a unique solution $h_{\varepsilon}$ to problem (1.15). Furthermore, $h_{\varepsilon}$ is increasing and concave in $[0,1]$, and there holds

$$
\mathcal{E}_{\varepsilon}\left(h_{\varepsilon}\right) \leq \frac{C_{1}}{\varepsilon}, \quad 0<h_{\varepsilon}^{\prime} \leq \frac{C_{1}}{\varepsilon},
$$

for some positive constant $C_{1}$ independent of $\varepsilon$.

The following lemma gives the main property of the profile function $h_{\varepsilon}$.

Lemma 4.2. For any $\varepsilon>0$ let $h_{\varepsilon}$ be the unique solution to problem (1.15), and still denote by $h_{\varepsilon}$ its odd reflection. Then (1.16) holds true.

Proof. Clearly we may assume $\tau \geq 0$. From (1.15) we get

$$
\frac{d}{d \tau}\left(\frac{1}{2} h_{\varepsilon}^{\prime 2}-F_{\varepsilon}\left(h_{\varepsilon}\right)\right)=h_{\varepsilon}^{\prime}\left[h_{\varepsilon}^{\prime \prime}-F_{\varepsilon}^{\prime}\left(h_{\varepsilon}\right)\right]=-\frac{\varphi^{\prime}}{\varphi} h_{\varepsilon}^{\prime 2} \leq 0 \quad \text { in }(0,1) .
$$


Still denote by $\varphi$ its even reflection. Since $h_{\varepsilon}$ is odd and $\varphi$ is even, $\varphi^{\prime}(\tau)>0$ for all $\tau>0$, from (4.2) we get

$$
\varepsilon\left(\frac{1}{2}\left[h_{\varepsilon}^{\prime}(\tau)\right]^{2}-F_{\varepsilon}\left(h_{\varepsilon}(\tau)\right)\right) \leq \varepsilon \frac{1}{2}\left[h_{\varepsilon}^{\prime}(1)\right]^{2}+\varepsilon \int_{|\tau|}^{1} \frac{\varphi^{\prime}}{\varphi} h_{\varepsilon}^{\prime 2} d s \quad \text { in }(-1,1) .
$$

Furthermore, using (4.1) we have

$$
\begin{aligned}
\varepsilon \int_{0}^{1} \frac{\varphi^{\prime}}{\varphi}\left(h^{\prime}\right)^{2} d s \leq & \left(\varepsilon \int_{0}^{1} \frac{\left(h^{\prime}\right)^{2}}{\varphi^{2}} d s\right)^{\frac{1}{2}}\left(\varepsilon \int_{0}^{1}\left(h^{\prime}\right)^{2} \varphi^{\prime 2} d s\right)^{\frac{1}{2}} \\
& \leq C_{1}\left(\varepsilon \int_{0}^{1}\left(\varphi^{\prime}\right)^{2}\left(h^{\prime}\right)^{2} d s\right)^{\frac{1}{2}}
\end{aligned}
$$

We shall prove that

$$
\left|h_{\varepsilon}^{\prime}\right| \leq \frac{o(1)}{\sqrt{\varepsilon}} \quad \text { if } \tau>\sqrt{\varepsilon}
$$

To this purpose, note that since $h_{\varepsilon}$ is concave in $(0,1)$, we have:

$$
0<h_{\varepsilon}^{\prime}(\tau) \leq \frac{1-h_{\varepsilon}(k \varepsilon)}{\sqrt{\varepsilon}-k \varepsilon} \text { for all } \tau \in[\sqrt{\varepsilon}, 1]
$$

for each $k \in(0, \infty)$ and for $\varepsilon \in(0,1)$ so small that $k \sqrt{\varepsilon}<1$. The function $v_{\varepsilon}(s):=h_{\varepsilon}(s \varepsilon)\left(s \in\left[0, \frac{1}{\epsilon}\right]\right)$ solves $v_{\varepsilon}^{\prime \prime}+\varepsilon \frac{\varphi^{\prime}(s \varepsilon)}{\varphi(s \varepsilon)} v_{\varepsilon}^{\prime}=f(v)$, and it is easy to see that it converges as $\varepsilon \rightarrow 0^{+}$in $C_{\text {loc }}^{2}(\mathbb{R})$ to a monotone increasing solution $v=v(s)$ of equation

$$
v^{\prime \prime}-f(v)=0 \quad \text { in }(-\infty, \infty)
$$

Now, note that

$$
\varepsilon^{2}\left\{\frac{1}{2} h_{\varepsilon}^{\prime}(\tau)^{2}-F_{\varepsilon}\left(h_{\varepsilon}(\tau)\right)\right\}=\varepsilon^{2} \int_{\tau}^{1} \frac{\varphi^{\prime}(s)}{\varphi(s)} h_{\varepsilon}^{\prime 2}(s) d s+\varepsilon^{2}\left\{\frac{1}{2} h_{\varepsilon}^{\prime}(1)^{2}-F_{\varepsilon}\left(h_{\varepsilon}(1)\right)\right\} .
$$

In view of the concavity of $h_{\varepsilon}$, it is easily seen that

$$
\varepsilon^{2}\left\{\frac{1}{2} h_{\varepsilon}^{\prime}(1)^{2}-F_{\varepsilon}\left(h_{\varepsilon}(1)\right)\right\} \rightarrow 0 \quad \text { as } \varepsilon \rightarrow 0^{+}
$$

while

$$
\varepsilon^{2}\left|\int_{\tau}^{1} \frac{\varphi^{\prime}(s)}{\varphi(s)} h_{\varepsilon}^{\prime 2}(s) d s\right| \leq C \varepsilon^{2} \mathcal{E}\left(h_{\varepsilon}\right) \leq C C_{1} \varepsilon
$$

in view of (4.1). Hence, from (4.2) we have

$$
\varepsilon^{2}\left\{\frac{1}{2} h_{\varepsilon}^{\prime}(\tau)^{2}-F_{\varepsilon}\left(h_{\varepsilon}(\tau)\right)\right\} \rightarrow 0 \quad \text { as } \varepsilon \rightarrow 0^{+} \text {for any } \tau \in[-1,1] .
$$


As a consequence,

$$
v_{\varepsilon}^{\prime}(0)=\varepsilon h_{\varepsilon}^{\prime}(0) \rightarrow \sqrt{2 F(0)}>0 \quad \text { as } \varepsilon \rightarrow 0^{+} .
$$

Thus, $v^{\prime}(0)=\sqrt{2 F(0)}>0, v$ is bounded and strictly increasing. Hence, in view of the well-known classification of bounded solutions to equation (4.7), $v(s) \rightarrow 1$ as $s \rightarrow \infty$, and $v(s) \rightarrow-1$ ad $s \rightarrow-\infty$. Since $h_{\varepsilon}(k \varepsilon)=v_{\varepsilon}(k) \rightarrow v(k)$ as $\varepsilon \rightarrow 0$, this combined with (4.6) gives (4.5).

Observe that, since $\varphi$ is smooth and $\varphi^{\prime}(0)=0$, there exists $C>0$ such that

$$
\varphi^{\prime}(\tau) \leq C \tau \quad \text { in }(0,1)
$$

Inequalities (4.4), (4.5) and (4.8) yield

$$
\begin{aligned}
\varepsilon \int_{0}^{1}\left(\varphi^{\prime}\right)^{2}\left(h_{\varepsilon}^{\prime}\right)^{2} d s & \leq C \varepsilon\left(\int_{0}^{\sqrt{\varepsilon}} \frac{s^{2}}{\varepsilon^{2}} d s+\int_{\sqrt{\varepsilon}}^{1} \frac{o(1)}{\varepsilon} d s\right) \\
& \leq C[\sqrt{\varepsilon}+o(1)] \rightarrow 0 \text { as } \varepsilon \rightarrow 0^{+}
\end{aligned}
$$

From (4.3), (4.4), (4.5) and (4.9) the conclusion follows.

\subsection{Well-prepared initial conditions}

Now we are ready to define well-prepared initial conditions $u_{0}^{\varepsilon}$. To be specific, we assume that the initial conditions $u_{0}^{\varepsilon}$ are in the form (1.18). In addition we assume that $u_{0}^{\varepsilon}$ and the corresponding $z_{0}^{\varepsilon} \equiv z^{\varepsilon}(\cdot, 0), \mu_{0}^{\varepsilon} \equiv \mu^{\varepsilon}(\cdot, 0)$ given by $(1.17),(1.13)$ satisfy

$\left(H_{1}^{*}\right) \quad\left\{\begin{array}{l}\text { (i) }\left(H_{1}\right) \text { is satisfied; } \\ \text { (ii) } z^{\varepsilon}(\cdot, 0) \in C^{2}(M) \text { and }\left|\nabla z^{\varepsilon}(x, 0)\right| \leq 1 \text { for all } x \in M .\end{array}\right.$

The construction of such a $u_{0}^{\varepsilon}$ is quite standard (see [36], [31]). Since we assume $\Sigma_{0}$ to be smooth (at least $C^{3}$ ), there is a small tubular neighboorod $U_{\delta} \supset \Sigma_{0}$ of size $4 \delta>0$ such that the distance function $d\left(x, \Sigma_{0}\right)$ is smooth in $U_{\delta}$ (at least $C^{2}$ ). Let now $\Psi \in C^{\infty}(\mathbb{R})$ an odd increasing function such that $\Psi(s)=s$ whenever $|s|<\delta$, $|\Psi(s)|=2 \delta$ for $|s| \geq 4 \delta$ and such that $\Psi^{\prime \prime} \leq 0$ for $s>0$. Then it is direct to see that if $\tilde{d}\left(x, \Sigma_{0}\right)$ is the signed distance from $\Sigma_{0}$, then $z^{\varepsilon}(x, 0)=\Psi\left(\tilde{d}\left(x, \Sigma_{0}\right)\right)$ is a globally smooth function (as smooth as the distance is near $\Sigma_{0}$ ), is constant far from $\Sigma_{0}$ and the corresponding $u_{0}^{\varepsilon}$ given by

$$
u_{0}^{\varepsilon}(x)=h_{\varepsilon}\left(\Psi\left(\tilde{d}\left(x, \Sigma_{0}\right)\right)\right)
$$

with $h^{\varepsilon}$ as in Lemma 4.1, satisfy assumption $\left(H_{1}^{*}\right)$ above. 
Let $\mathcal{F}(s):=\int_{0}^{s} \sqrt{F(\tau)} d \tau$. We have that $u_{0}^{\varepsilon} \rightarrow u_{0}^{0}$, and $\mathcal{F}\left(u_{0}^{\varepsilon}\right) \rightarrow \mathcal{F}\left(u_{0}^{0}\right)$ as $\varepsilon \rightarrow 0^{+}$, uniformly in $M \backslash \Sigma_{0}$ and in $L_{\text {loc }}^{1}(M)$; furthermore, for $c=\int_{-1}^{1} \sqrt{2 F(s)} d s$,

$$
\int_{\Omega}\left(\frac{\varepsilon}{2}\left|\nabla u_{0}^{\varepsilon}\right|^{2}+\frac{F\left(u_{0}^{\varepsilon}\right)}{\varepsilon}\right) d \mathcal{V} \rightarrow c\left|\nabla u_{0}^{0}\right|(\Omega) \quad \text { as } \varepsilon \rightarrow 0^{+}, \text {for every } \Omega \Subset M,
$$

where $\left|\nabla u_{0}^{0}\right|(\Omega)$ denotes the total variation of $u_{0}^{0}$ in $\Omega$, whence $\mathcal{F}\left(u_{0}^{\varepsilon}\right) \rightarrow \mathcal{F}\left(u_{0}^{0}\right)$ as $\varepsilon \rightarrow 0^{+}$weakly-* in $B V_{\mathrm{loc}}(M)$.

Note that if $\left(H_{0}\right),\left(H_{1}^{*}\right)$ are satisfied, then the unique solution to problem (1.1)(1.2), which exists by Proposition 5.9, also verifies

$$
-1<u^{\varepsilon}<1 \text { in } M \times(0, \infty) .
$$

This follows by the maximum principle, since $\left|u_{0}^{\varepsilon}\right| \leq 1$. In addition, since we assume $u_{0}^{\varepsilon} \in C^{2}(M)$, parabolic regularity theory also implies $\nabla u^{\varepsilon} \in C^{0}(M \times$ $[0, \infty))$

\subsection{Asymptotic control of discrepancy}

In order to show (1.14) we need to prove preliminarily that (1.21) follows from $\left(H_{0}\right),\left(H_{1}^{*}\right)$.

Lemma 4.3. Let assumptions $\left(H_{0}\right),\left(H_{1}^{*}\right)$ be satisfied. Let $u^{\varepsilon}$ be a solution to equation (1.1); suppose that (1.19) and (1.20) hold true. Then inequality (1.21) is satisfied.

Proof. Define $w^{\varepsilon}:=\left|\nabla z^{\varepsilon}\right|^{2}$, and note that, as already proved above, $w^{\varepsilon} \in C^{0}(M \times$ $[0, \infty) \cap \cap C^{\infty}(M \times(0, \infty))$. From (1.1), (1.12), (2.7), (1.15) and (1.20) we deduce that

$$
\begin{aligned}
\partial_{t} w^{\varepsilon} \leq & \Delta w^{\varepsilon}-2\left|\operatorname{Hess} z^{\varepsilon}\right|^{2}-2 \lambda w^{\varepsilon}-\frac{f\left(u^{\varepsilon}\right)}{\varepsilon^{2} h_{\varepsilon}^{\prime}\left(z^{\varepsilon}\right)}\left\langle\nabla z^{\varepsilon}, \nabla w^{\varepsilon}\right\rangle \\
& -\frac{w^{\varepsilon}}{\varepsilon^{2}}\left(w^{\varepsilon}-1\right)\left[f^{\prime}\left(u^{\varepsilon}\right)-f\left(u^{\varepsilon}\right) \frac{h^{\prime \prime}\left(z^{\varepsilon}\right)}{\left(h^{\prime}\left(z^{\varepsilon}\right)\right)^{2}}\right] \\
& -\frac{\varphi^{\prime}}{\varphi}\left(z^{\varepsilon}\right)\left\langle\nabla z^{\varepsilon}, \nabla w^{\varepsilon}\right\rangle-\left(\frac{\varphi^{\prime}}{\varphi}\right)^{\prime}\left(z^{\varepsilon}\right) w^{\varepsilon} \\
\leq & \Delta w^{\varepsilon}-\frac{f\left(u^{\varepsilon}\right)}{\varepsilon^{2} h_{\varepsilon}^{\prime}\left(z^{\varepsilon}\right)}\left\langle\nabla z^{\varepsilon}, \nabla w^{\varepsilon}\right\rangle \\
& -\frac{w^{\varepsilon}}{\varepsilon^{2}}\left(w^{\varepsilon}-1\right)\left[f^{\prime}\left(u^{\varepsilon}\right)-f\left(u^{\varepsilon}\right) \frac{h^{\prime \prime}\left(z^{\varepsilon}\right)}{\left(h^{\prime}\left(z^{\varepsilon}\right)\right)^{2}}\right] \text { in } M \times(0, \infty) .
\end{aligned}
$$

Thus, $w^{\varepsilon}$ is a subsolution to the equation

$$
\begin{aligned}
\partial_{t} w^{\varepsilon}= & \Delta w^{\varepsilon}-\frac{f\left(u^{\varepsilon}\right)}{\varepsilon^{2} h_{\varepsilon}^{\prime}\left(z^{\varepsilon}\right)}\left\langle\nabla z^{\varepsilon}, \nabla w^{\varepsilon}\right\rangle \\
& -\frac{w^{\varepsilon}}{\varepsilon^{2}}\left(w^{\varepsilon}-1\right)\left[f^{\prime}\left(u^{\varepsilon}\right)-f\left(u^{\varepsilon}\right) \frac{h^{\prime \prime}\left(z^{\varepsilon}\right)}{\left(h^{\prime}\left(z^{\varepsilon}\right)\right)^{2}}\right] \quad \text { in } M \times(0, \infty) .
\end{aligned}
$$


On the other hand, the function $\bar{w} \equiv 1$ is a solution to equation (4.12). Note that since (1.12) holds true, from results in [17] (where (1.12) is assumed) comparison principles can be easily obtained. Hence, from (1.19) and comparison principles inequality (1.21) follows.

Now we can prove the following:

Proposition 4.4. Let the assumptions of Lemma 4.3 be satisfied. Then

$$
\limsup _{\varepsilon \rightarrow 0^{+}} \sup _{(x, t) \in M \times(0, \infty)} \xi_{t}^{\varepsilon}(x) \leq 0 .
$$

Hence, in particular, (1.14) holds true.

Proof. From (4.2) and (1.21), we get

$$
\begin{aligned}
\frac{1}{2}\left|\nabla u^{\varepsilon}\right|^{2}-F_{\varepsilon}\left(u^{\varepsilon}\right) & =\frac{1}{2}\left[h_{\varepsilon}^{\prime}\left(z^{\varepsilon}\right)\right]^{2}\left|\nabla z^{\varepsilon}\right|^{2}-F_{\varepsilon}\left(h_{\varepsilon}\left(z^{\varepsilon}\right)\right) \\
& \leq \frac{1}{2}\left[h_{\varepsilon}^{\prime}\left(z^{\varepsilon}\right)\right]^{2}-F_{\epsilon}\left(h_{\epsilon}\left(z^{\epsilon}\right)\right) \quad \text { in } M \times(0, \infty) .
\end{aligned}
$$

From Lemma 4.2 the conclusion follows.

\section{Uniform energy bounds}

This section is devoted to proving the local almost monotonicity formula (1.22) and to derive from a priori estimates some compactness properties of the family of solutions $u^{\varepsilon}$ as $\varepsilon \rightarrow 0$ both in $B V_{\text {loc }}$ and in $L_{\text {loc }}^{1}$.

\subsection{Local almost monotonicity formula}

The argument to prove (1.22) is a modification of the one in [31], localizing the estimate at suitably small scale so to reabsorbe the perturbation terms coming from the curved background. This, combined with the locally uniform control of the positive part of the discrepancy from the previous sections, will give the final result.

First, recall Lemma 6.6 in [32]:

Lemma 5.1. Let $\varphi \in C_{c}^{2}(M ;[0, \infty))$. Then

$$
\frac{|\nabla \varphi|^{2}}{\varphi} \leq 2 \max _{\{\varphi>0\}}|\operatorname{Hess} \varphi| \quad \text { in }\{\varphi>0\} .
$$

Then, the next lemma will give a a sort of Brakke's inequality for $d \mu_{t}^{\varepsilon}$. 
Lemma 5.2. Let $u^{\varepsilon}$ be a solution to equation (1.1). Let $\phi \in C_{x, t}^{2,1}\left(M \times(0, \infty) ; \mathbb{R}_{+}\right)$ with supp $\phi(\cdot, t)$ compact for every $t \in(0, \infty)$. Then

$$
\begin{aligned}
& \frac{d}{d t} \int_{M} \phi E^{\varepsilon} d \mathcal{V}(x) d t \\
& =\int_{M}\left\{\left(\partial_{t} \phi-\Delta \phi\right) E^{\varepsilon}+(\operatorname{Hess} \phi)\left(\nabla u^{\varepsilon}, \nabla u^{\varepsilon}\right)-\phi\left(\partial_{t} u^{\varepsilon}\right)^{2}\right\} d \mathcal{V}(x),
\end{aligned}
$$

and

$$
\begin{aligned}
& \frac{d}{d t} \int_{M} \phi E^{\varepsilon} d \mathcal{V}(x) d t \\
& =\int_{M}\left\{\left(\partial_{t} \phi+\Delta \phi\right) E^{\varepsilon}-(\operatorname{Hess} \phi)\left(\nabla u^{\varepsilon}, \nabla u^{\varepsilon}\right)\right\} d \mathcal{V}(x) \\
& \quad+\int_{M} \frac{\left\langle\nabla \phi, \nabla u^{\varepsilon}\right\rangle^{2}}{\phi} d \mathcal{V}(x)-\int_{M} \phi\left(\partial_{t} u^{\varepsilon}+\frac{\left\langle\nabla \phi, \nabla u^{\varepsilon}\right\rangle}{\phi}\right)^{2} d \mathcal{V}(x) .
\end{aligned}
$$

for all $t>0$.

Note that the last two integrals in equality (5.2) are well-defined, in view of Lemma 5.1.

Proof. By $(1.1)$, in $M \times(0, \infty)$,

$$
\phi \partial_{t} E^{\varepsilon}=\phi\left\langle\nabla \partial_{t} u^{\varepsilon}, \nabla u^{\varepsilon}\right\rangle+\phi \partial_{t} u^{\varepsilon} \Delta u^{\varepsilon}-\phi\left(\partial_{t} u^{\varepsilon}\right)^{2},
$$

and

$$
\begin{aligned}
\left\langle\nabla \phi, \nabla E^{\varepsilon}\right\rangle= & \frac{1}{2}\left\langle\nabla \phi, \nabla\left\langle\nabla u^{\varepsilon}, \nabla u^{\varepsilon}\right\rangle\right\rangle \\
& -\partial_{t} u^{\varepsilon}\left\langle\nabla \phi, \nabla u^{\varepsilon}\right\rangle+\left\langle\nabla u^{\varepsilon}, \nabla \phi\right\rangle \Delta u^{\varepsilon}
\end{aligned}
$$

In view of (5.3)-(5.4) we get

$$
\begin{aligned}
\phi \partial_{t} E^{\varepsilon}-\left\langle\nabla \phi, \nabla E^{\varepsilon}\right\rangle= & \phi\left\langle\nabla \partial_{t} u^{\varepsilon}, \nabla u^{\varepsilon}\right\rangle+\phi \partial_{t} u^{\varepsilon} \Delta u^{\varepsilon}-\phi\left(\partial_{t} u^{\varepsilon}\right)^{2} \\
& -\frac{1}{2}\left\langle\nabla \phi, \nabla\left\langle\nabla u^{\varepsilon}, \nabla u^{\varepsilon}\right\rangle\right\rangle \\
& +\partial_{t} u^{\varepsilon}\left\langle\nabla \phi, \nabla u^{\varepsilon}\right\rangle-\left\langle\nabla u^{\varepsilon}, \nabla \phi\right\rangle \Delta u^{\varepsilon}
\end{aligned}
$$


From (5.5), integrating by parts and using (2.6), it follows:

$$
\begin{aligned}
\frac{d}{d t} \int_{M} \phi E^{\varepsilon} d \mathcal{V}(x)= & \int_{M} \partial_{t} \phi E^{\varepsilon} d \mathcal{V}(x)+\int_{M}\left\{\left\langle\nabla \phi, \nabla E^{\varepsilon}\right\rangle-\phi\left(\partial_{t} u^{\varepsilon}\right)^{2}\right. \\
& +\phi \partial_{t} u^{\varepsilon} \Delta u^{\varepsilon}+\phi\left\langle\nabla \partial_{t} u^{\varepsilon}, \nabla u^{\varepsilon}\right\rangle-\frac{1}{2}\left\langle\nabla \phi, \nabla\left\langle\nabla u^{\varepsilon}, \nabla u^{\varepsilon}\right\rangle\right\rangle \\
& \left.+\partial_{t} u^{\varepsilon}\left\langle\nabla \phi, \nabla u^{\varepsilon}\right\rangle-\Delta u^{\varepsilon}\left\langle\nabla \phi, \nabla u^{\varepsilon}\right\rangle\right\} d \mathcal{V}(x) \\
= & \int_{M}\left(\partial_{t} \phi-\Delta \phi\right) E^{\varepsilon} d \mathcal{V}(x)-\int_{M} \phi\left(\partial_{t} u^{\varepsilon}\right)^{2} d \mathcal{V}(x) \\
& -\int_{M}\left\{\frac{1}{2}\left\langle\nabla \phi, \nabla\left\langle\nabla u^{\varepsilon}, \nabla u^{\varepsilon}\right\rangle\right\rangle-\left\langle\nabla u^{\varepsilon}, \nabla\left\langle\nabla \phi, \nabla u^{\varepsilon}\right\rangle\right\rangle\right\} d \mathcal{V}(x) \\
= & \int_{M}\left(\partial_{t} \phi-\Delta \phi\right) E^{\varepsilon} d \mathcal{V}(x)-\int_{M} \phi\left(\partial_{t} u^{\varepsilon}\right)^{2} d \mathcal{V}(x) \\
& -\int_{M}\left\{\frac{1}{2} \operatorname{Hess}\left(u^{\varepsilon}\right)\left\langle\nabla u^{\varepsilon}, \nabla \phi\right\rangle+\frac{1}{2} \operatorname{Hess}^{\varepsilon}\left(u^{\varepsilon}\right)\left\langle\nabla u^{\varepsilon}, \nabla \phi\right\rangle\right. \\
& \left.-\operatorname{Hess}^{\varepsilon}\left(u^{\varepsilon}\right)\left(\nabla u^{\varepsilon}, \nabla \phi\right)+\operatorname{Hess}(\phi)\left(\nabla u^{\varepsilon}, \nabla u^{\varepsilon}\right)\right\} d \mathcal{V}(x) \\
= & \int_{M}\left(\partial_{t} \phi-\Delta \phi\right) E^{\varepsilon} d \mathcal{V}(x)-\int_{M} \phi\left(\partial_{t} u^{\varepsilon}\right)^{2} d \mathcal{V}(x) \\
& +\int_{M} \operatorname{Hess}(\phi)\left(\nabla u^{\varepsilon}, \nabla u^{\varepsilon}\right) d \mathcal{V}(x) .
\end{aligned}
$$

Hence (5.1) has been verified. Equality (5.2) can be shown analogously, using

$$
\begin{aligned}
\phi \partial_{t} E^{\varepsilon}+\left\langle\nabla \phi, \nabla E^{\varepsilon}\right\rangle= & \phi\left\langle\nabla \partial_{t} u^{\varepsilon}, \nabla u^{\varepsilon}\right\rangle+\phi \partial_{t} u^{\varepsilon} \Delta u^{\varepsilon}-\phi\left(\partial_{t} u^{\varepsilon}\right)^{2} \\
& +\frac{1}{2}\left\langle\nabla \phi, \nabla\left\langle\nabla u^{\varepsilon}, \nabla u^{\varepsilon}\right\rangle\right\rangle-\partial_{t} u^{\varepsilon}\left\langle\nabla \phi, \nabla u^{\varepsilon}\right\rangle \\
& +\left\langle\nabla u^{\varepsilon}, \nabla \phi\right\rangle \Delta u^{\varepsilon},
\end{aligned}
$$

instead of (5.5).

Next we are going to choose a precise test function in the formulas obtained above. The following lemma gives auxiliary identities which will be useful in this direction.

Lemma 5.3. Let $K \subset M$ be a compact subset, $y \in K, s>0$. Let $\hat{\zeta} \in C^{2}([0, \infty))$ such that

$$
\begin{gathered}
|\hat{\zeta}| \leq 1, \quad\left|\hat{\zeta}^{\prime}\right| \leq 1,\left|\hat{\zeta}^{\prime \prime}\right| \leq 1 \text { in }[0, \infty), \\
\hat{\zeta}=\left\{\begin{array}{l}
1 \text { in }\left[0, R_{0}^{2} / 4\right) \\
0 \text { in }\left[R_{0}^{2}, \infty\right),
\end{array}\right.
\end{gathered}
$$


where $R_{0}:=\frac{1}{2} \inf _{y \in K} \operatorname{inj}(y)$. Define

$$
\hat{\eta}(\rho, t):=[(s-t)]^{-\frac{N-1}{2}} e^{-\frac{\rho}{4(s-t)}} \quad(\rho \geq 0,0 \leq t<s) .
$$

Let

$$
\begin{gathered}
\eta(x, t):=\hat{\eta}\left(d^{2}(x), t\right) \quad(x \in M, 0 \leq t<s) \\
\zeta(x):=\hat{\zeta}\left(d^{2}(x)\right) \quad(x \in M) .
\end{gathered}
$$

Then, for all $x \in M, 0 \leq t<s$,

$$
\begin{aligned}
\nabla(\eta \zeta)(x, t)= & \partial_{\rho}(\hat{\eta} \hat{\zeta})\left(d^{2}(x), t\right) \nabla d^{2}(x) \\
\operatorname{Hess}[(\eta \zeta)]\left(d^{2}(x), t\right)(X, X)= & \partial_{\rho \rho}(\hat{\eta} \hat{\zeta})\left(d^{2}(x), t\right)\left|\left\langle\nabla d^{2}(x), X\right\rangle\right|^{2} \\
& +\partial_{\rho}(\hat{\eta} \hat{\zeta})\left(d^{2}(x), t\right) \operatorname{Hess}\left[d^{2}(x)\right](X, X) \\
\Delta(\eta \zeta)(x, t)= & \partial_{\rho \rho}(\hat{\eta} \hat{\zeta})\left(d^{2}(x), t\right)\left|\nabla d^{2}(x)\right|^{2} \\
& +\left(\partial_{\rho} \hat{\eta} \hat{\zeta}\right)\left(d^{2}(x), t\right) \Delta d^{2}(x) ; \\
\left(\partial_{t}+\Delta\right)(\eta \zeta)(x, t)= & \frac{(\eta \zeta)(x, t)}{s-t}\left[\frac{N-1}{2}-\frac{d^{2}(x)}{4(s-t)}\right. \\
& +\left(2 \partial_{\rho} \hat{\eta} \partial_{\rho} \hat{\zeta}+\hat{\eta} \partial_{\rho \rho} \hat{\zeta}\right)\left(d^{2}(x), t\right)\left|\nabla d^{2}(x)\right|^{2} \\
& +\left(\hat{\eta} \partial_{\rho} \hat{\zeta}\right)\left(d^{2}(x), t\right) \Delta d^{2}(x)
\end{aligned}
$$

Remark 5.4. It is straightforward to check that

$$
\partial_{\rho} \hat{\eta}=-\frac{\hat{\eta}}{4(s-t)}, \quad \partial_{\rho \rho} \frac{\hat{\eta}}{16(s-t)^{2}}, \quad\left(\partial_{\rho} \hat{\eta}\right)^{2}-\hat{\eta} \partial_{\rho \rho} \hat{\eta}=0 .
$$

Proof of Lemma 5.3. For any $x \in M, 0 \leq t<s$ we have

$$
\nabla(\eta \zeta)(x, t)=\partial_{\rho}(\hat{\eta} \hat{\zeta})\left(d^{2}(x), t\right) \nabla d^{2}(x)
$$

Hence, for any $X \in T_{x} M$,

$$
\begin{aligned}
\operatorname{Hess}[(\eta \zeta)](x, t)(X, X)= & \left\langle\mathrm{D}_{X} \nabla(\eta \zeta), X\right\rangle=\left\langle\mathrm{D}_{X}\left(\partial_{\rho} \hat{\eta} \hat{\zeta}\right)\left(d^{2}(x), t\right) \nabla d^{2}(x), X\right\rangle \\
= & \partial_{\rho \rho}(\hat{\eta} \hat{\zeta})\left(d^{2}(x), t\right)\left|\left\langle X, \nabla d^{2}(x)\right\rangle\right|^{2} \\
& +\partial_{\rho}(\hat{\eta} \hat{\zeta})\left(d^{2}(x), t\right) \operatorname{Hess}\left[d^{2}(x)\right](X, X) .
\end{aligned}
$$


Passing to the trace we get

$$
\Delta(\eta \zeta)(x, t)=\partial_{\rho \rho}(\hat{\eta} \hat{\zeta})\left(d^{2}(x), t\right)\left|\nabla d^{2}(x)\right|^{2}+\partial_{\rho}(\hat{\eta} \hat{\zeta})\left(d^{2}(x), t\right) \Delta d^{2}(x) .
$$

Furthermore,

$$
\partial_{t} \hat{\eta}=-\frac{1-N}{2} \frac{\hat{\eta}}{s-t}+\hat{\eta}\left(-\frac{\rho}{4(s-t)^{2}}\right)=\frac{\hat{\eta}}{s-t}\left[\frac{N-1}{2}-\frac{\rho}{4(s-t)}\right] .
$$

Note that

$$
\left(\partial_{t}+\Delta\right)(\eta \zeta)(x, t)=\zeta\left(\partial_{t}+\Delta\right) \eta(x, t)+2 \nabla \eta(x, t) \nabla \zeta(x, t)+\eta \Delta \zeta(x) ;
$$

moreover,

$$
\nabla \eta(x, t)=\partial_{\rho} \hat{\eta}\left(d^{2}(x), t\right) \nabla d^{2}(x)
$$

and

$$
\Delta \eta(x, t)=\partial_{\rho \rho} \hat{\eta}\left(d^{2}(x), t\right)\left|\nabla d^{2}(x)\right|^{2}+\partial_{\rho} \hat{\eta}\left(d^{2}(x), t\right) \Delta d^{2}(x) .
$$

By (5.15)-(5.17),

$$
\left(\partial_{t}+\Delta\right) \eta(x, t)=\frac{\hat{\eta}}{s-t}\left[\frac{N-1}{2}-\frac{d^{2}(x)}{4(s-t)}+\frac{\left|\nabla d^{2}(x)\right|^{2}}{16(s-t)}-\frac{1}{4} \Delta d^{2}(x)\right],
$$

so

$$
\begin{aligned}
& \left(\partial_{t}+\Delta\right)(\eta \zeta)(x, t) \\
& =\frac{(\hat{\eta} \hat{\zeta})\left(d^{2}(x), t\right)}{s-t}\left[-\frac{1}{2}+\frac{d^{2}(x)}{4(s-t)}\left(-1+|\nabla d(x)|^{2}\right)+\frac{N}{2}-\frac{1}{4} \Delta d^{2}(x)\right] \\
& \quad+\left(2 \partial_{\rho} \hat{\eta} \partial_{\rho} \hat{\zeta}+\hat{\eta} \partial_{\rho \rho} \hat{\zeta}\right)\left(d^{2}(x), t\right)\left|\nabla d^{2}(x)\right|^{2}+\left(\hat{\eta} \partial_{\rho} \hat{\zeta}\right)\left(d^{2}(x), t\right) \Delta d^{2}(x) .
\end{aligned}
$$

This implies (5.12), since $|\nabla d(x)|^{2} \leq 1$. This completes the proof.

In order to proceed we start rewriting some terms in (5.2). Note that

$$
\begin{aligned}
& \frac{\left|\left\langle\nabla \phi, \nabla u^{\varepsilon}\right\rangle\right|^{2}}{\phi}-\operatorname{Hess} \phi\left(\nabla u^{\varepsilon}, \nabla u^{\varepsilon}\right) \\
& =\frac{\left|\left\langle\nabla d^{2}(x), \nabla u^{\varepsilon}\right\rangle\right|^{2}}{\eta \zeta}\left(\hat{\zeta} \partial_{\rho} \hat{\eta}+\hat{\eta} \partial_{\rho} \hat{\zeta}\right)^{2}-\left(\hat{\zeta} \partial_{\rho} \hat{\eta}+\hat{\eta} \partial_{\rho} \hat{\zeta}\right) \operatorname{Hess}\left[d^{2}(x)\right]\left(\nabla u^{\varepsilon}, \nabla u^{\varepsilon}\right) \\
& \quad-\left|\left\langle\nabla d^{2}(x), \nabla u^{\varepsilon}\right\rangle\right|^{2}\left(\hat{\zeta} \partial_{\rho \rho} \hat{\eta}+2 \partial_{\rho} \hat{\zeta} \partial_{\rho} \hat{\eta}+\hat{\eta} \partial_{\rho \rho} \hat{\zeta}\right) \\
& =\frac{\hat{\eta} \hat{\zeta}}{4(s-t)} \operatorname{Hess}\left[d^{2}(x)\right]\left(\nabla u^{\varepsilon}, \nabla u^{\varepsilon}\right)-\hat{\eta} \partial_{\rho} \hat{\zeta} \operatorname{Hess}\left[d^{2}(x)\right]\left(\nabla u^{\varepsilon}, \nabla u^{\varepsilon}\right) \\
& +\left|\left\langle\nabla d^{2}(x), \nabla u^{\varepsilon}\right\rangle\right|^{2}\left[\frac{\hat{\zeta}^{2}\left(\partial_{\rho} \hat{\eta}\right)^{2}+2 \hat{\eta} \partial_{\rho} \hat{\eta} \hat{\zeta} \partial_{\rho} \hat{\zeta}+\hat{\eta}^{2}\left(\partial_{\rho} \hat{\zeta}\right)^{2}}{\hat{\eta} \hat{\zeta}}\right. \\
& \left.\quad-\hat{\zeta} \partial_{\rho \rho} \hat{\eta}-2 \partial_{\rho} \hat{\eta} \partial_{\rho} \hat{\zeta}-\hat{\eta} \partial_{\rho \rho} \hat{\zeta}\right] .
\end{aligned}
$$


So, we can infer the following:

Lemma 5.5. Let $\eta$ and $\zeta$ be as in Lemma 5.3, and $\phi:=\eta \zeta$. Then

$$
\begin{aligned}
& \int_{M}\left[\varepsilon \frac{\left|\left\langle\nabla \phi, \nabla u^{\varepsilon}\right\rangle\right|^{2}}{\phi}-\varepsilon \operatorname{Hess} \phi\left(\nabla u^{\varepsilon}, \nabla u^{\varepsilon}\right)\right] d \mathcal{V}(x) \\
& =\varepsilon \int_{M} \frac{\eta \zeta}{2(s-t)}\left|\nabla u^{\varepsilon}\right|^{2} d \mathcal{V}(x) \\
& \quad+\varepsilon \int_{M} \frac{\eta \zeta}{2(s-t)}\left[\frac{1}{2} \operatorname{Hess}\left[d^{2}(x)\right]\left(\nabla u^{\varepsilon}, \nabla u^{\varepsilon}\right)-\left|\nabla u^{\varepsilon}\right|^{2}\right] d \mathcal{V}(x) \\
& \quad+\varepsilon \int_{M}\left|\left\langle\nabla d^{2}(x), \nabla u^{\varepsilon}\right\rangle\right|^{2} \eta\left(\frac{\partial_{\rho} \hat{\zeta}}{\hat{\zeta}}-\partial_{\rho \rho} \hat{\zeta}\right) d \mathcal{V}(x) \\
& \quad-\varepsilon \int_{M} \eta \partial_{\rho} \hat{\zeta} \operatorname{Hess}\left[d^{2}(x)\right]\left(\nabla u^{\varepsilon}, \nabla u^{\varepsilon}\right) d \mathcal{V}(x) .
\end{aligned}
$$

From Lemmas 5.2, 5.3, 5.5, neglecting one negative term we immediately get:

Lemma 5.6. Let $\phi:=\eta \zeta$. Then

$$
\begin{aligned}
\frac{d}{d t} \int_{M} \phi \varepsilon E^{\varepsilon} d \mathcal{V}(x) \leq & \int_{M} \frac{\phi}{2(s-t)}\left[\varepsilon\left|\nabla u^{\varepsilon}\right|^{2}-\varepsilon E^{\varepsilon}\right] d \mathcal{V}(x) \\
& +\varepsilon \int_{M} \frac{\phi}{2(s-t)}\left[\frac{1}{2} \operatorname{Hess}\left[d^{2}(x)\right]\left(\nabla u^{\varepsilon}, \nabla u^{\varepsilon}\right)-\left|\nabla u^{\varepsilon}\right|^{2}\right] d \mathcal{V}(x) \\
& +\varepsilon \int_{M} \frac{\phi}{2(s-t)}\left[N-\frac{1}{2} \Delta d^{2}(x)\right] E^{\varepsilon} d \mathcal{V}(x) \\
& +\varepsilon \int_{M} E^{\varepsilon} \eta \partial_{\rho} \hat{\zeta} \Delta d^{2}(x) \\
& +\varepsilon \int_{M} E^{\varepsilon}\left|\nabla d^{2}(x)\right|^{2}\left(2 \partial_{\rho} \hat{\eta} \partial_{\rho} \hat{\zeta}+\hat{\eta} \partial_{\rho \rho} \hat{\zeta}\right) d \mathcal{V}(x) \\
& -\varepsilon \int_{M} \eta \partial_{\rho} \hat{\zeta} \operatorname{Hess}\left[d^{2}(x)\right]\left(\nabla u^{\varepsilon}, \nabla u^{\varepsilon}\right) d \mathcal{V}(x) \\
& +\varepsilon \int_{M}\left|\left\langle\nabla d^{2}(x), \nabla u^{\varepsilon}\right\rangle\right|^{2} \eta\left(\frac{\left(\partial_{\rho} \hat{\zeta}\right)^{2}}{\hat{\zeta}}-\partial_{\rho \rho} \hat{\zeta}\right) d \mathcal{V}(x)
\end{aligned}
$$

Finally, we single out the discrepancy term and enstimate all the others to obtain the key result of this section.

Proposition 5.7. Let assumption $\left(H_{0}\right)$ be satisfied. Let $u^{\varepsilon}$ be the solution to problem (1.1)-(1.2). Suppose that (3.3) and (3.8) with $\tau=0$ hold true. Let $K \subset M$ be 
a compact subset, $y \in K, s>0$. Let $\phi:=\eta \zeta$ with $\eta$ and $\zeta$ as in Lemma 5.3. Then for every $\varepsilon \in(0,1)$

$$
\begin{aligned}
\frac{d}{d t} \int_{M} \phi(x, t) d \mu_{t}^{\varepsilon}(x) \leq & \frac{1}{2(s-t)} \int_{M} \phi \varepsilon\left\{\left|\nabla u^{\varepsilon}\right|^{2}-E^{\varepsilon}\right\} d \mathcal{V}(x) \\
& +\frac{C_{3}}{(s-t)^{1 / 2}} \int_{M} \phi d \mu_{t}^{\varepsilon}(x)+C_{4} \text { for all } 0<t<s
\end{aligned}
$$

for some positive constants $C_{3}$ and $C_{4}$ depending only on $K$.

Proof. By Lemma 3.5 and Lemma 5.6,

$$
\begin{aligned}
\frac{d}{d t} \int_{M} \phi \varepsilon E^{\varepsilon} d \mathcal{V}(x) \leq & \frac{1}{2(s-t)} \int_{M} \phi d \xi_{t}^{\varepsilon}+C \int_{M} \phi \frac{d^{2}(x)}{s-t} \varepsilon E^{\varepsilon} d \mathcal{V}(x) \\
& +C_{R_{0}} \int_{M} \varepsilon E^{\varepsilon} \eta\left(\left|\partial_{\rho} \hat{\zeta}\right|+\frac{\left|\partial_{\rho} \hat{\zeta}\right|}{\hat{\zeta}}+\left|\partial_{\rho \rho} \hat{\zeta}\right|\right) d \mathcal{V}(x) \\
& +C_{R_{0}} \int_{M} \varepsilon E^{\varepsilon} \eta\left(\frac{d^{2}(x)}{s-t}\left|\partial_{\rho} \hat{\zeta}\right|+\left|\partial_{\rho \rho} \hat{\zeta}\right|\right) d \mathcal{V}(x) \\
\leq & \frac{1}{2(s-t)} \int_{M} \phi d \xi_{t}^{\varepsilon}+C \int_{M} \phi \frac{d^{2}(x)}{s-t} \varepsilon E^{\varepsilon} d \mathcal{V}(x) \\
& +\tilde{C}_{R_{0}} \int_{\frac{R_{0}}{2} \leq d(x) \leq R_{0}} \varepsilon E^{\varepsilon} d \mathcal{V}(x)
\end{aligned}
$$

for some positive constants $C, C_{R_{0}}, \tilde{C}_{R_{0}}$ independent of $\varepsilon$.

Now, note that

$$
\begin{aligned}
\int_{M} \phi \frac{d^{2}(x)}{s-t} \varepsilon E^{\varepsilon} d \mathcal{V}(x) \leq & \int_{B_{R_{0}}(y)} \phi \frac{d^{2}(x)}{s-t} \varepsilon E^{\varepsilon} d \mathcal{V}(x) \\
\leq & \int_{B_{R_{0}} \cap\{d(x)>\sqrt[4]{s-t}\}} \zeta \eta \frac{d^{2}(x)}{s-t} \varepsilon E^{\varepsilon} d \mathcal{V}(x) \\
& +\int_{B_{R_{0}} \cap\{d(x)<\sqrt[4]{s-t}\}} \zeta \eta \frac{d^{2}(x)}{s-t} \varepsilon E^{\varepsilon} d \mathcal{V}(x) .
\end{aligned}
$$

Furthermore, in view of (3.8), there exists a positive constant $\underline{C}_{K}$, independent of $\varepsilon$, such that

$$
\mu_{t}^{\varepsilon}(K) \leq \underline{C}_{K} .
$$

We have

$$
\int_{B_{R_{0}} \cap\{d(x)<\sqrt[4]{s-t}\}} \zeta \eta \frac{d^{2}(x)}{s-t} \varepsilon E^{\varepsilon} d \mathcal{V}(x) \leq \frac{\tilde{C}}{\sqrt{s-t}} \int_{M} \phi \varepsilon E^{\varepsilon} d \mathcal{V}(x)
$$


moreover, since $B_{R_{0}}(y) \subset K$, from (5.21) we can infer that

$$
\begin{aligned}
& \int_{B_{R_{0}} \cap\{d(x)>\sqrt[4]{s-t}\}} \zeta \eta \frac{d^{2}(x)}{s-t} \varepsilon E^{\varepsilon} d \mathcal{V}(x) \\
& \leq \underline{C} \int_{B_{R_{0}} \cap\{d(x)>\sqrt[4]{s-t}\}} \frac{d^{2}(x)}{(s-t)^{\frac{N+1}{2}}} e^{-\frac{d^{2}(x)}{4(s-t)}} \varepsilon E^{\varepsilon} d \mathcal{V}(x) \\
& \leq \underline{C} \int_{B_{R_{0}} \cap\{d(x)>\sqrt[4]{s-t}\}} \frac{d^{2}(x)}{s-t} e^{-\frac{d^{2}(x)}{8(s-t)}} \frac{e^{-\frac{1}{4 \sqrt{s-t}}}}{(s-t)^{\frac{N-1}{2}}} \varepsilon E^{\varepsilon} d \mathcal{V}(x) \leq \bar{C}_{K},
\end{aligned}
$$

for some positive constants $\tilde{C}, \underline{C}, \bar{C}_{K}$ independent of $\varepsilon$.

On the other hand, observe that the functions $(x, t) \mapsto \eta(x, t)$ and $(x, t) \mapsto$ $\frac{\eta(x, t)}{s-t}$ are bounded in $\left.\left\{\frac{R_{0}}{2} \leq d(x) \leq R_{0}\right\} \times(0, s)\right)$; thus

$$
\int_{\left\{\frac{R_{0}}{2} \leq d(x) \leq R_{0}\right\}} \varepsilon E^{\varepsilon} d \mathcal{V}(x) \leq \check{C}_{K},
$$

for some positive constant $\check{C}_{K}$ independent of $\varepsilon$.

From (5.19), (5.20)-(5.23) we obtain (5.18), with

$$
C_{3}=C \tilde{C}_{K}, \quad C_{4}=\max \left\{C \bar{C}_{K}, \tilde{C}_{R_{0}} \check{C}_{K}\right\}
$$

From Lemma 5.7 and the asymptotic control of discrepancy in Proposition 3.4 we finally deduce the main result of this section.

Theorem 5.8. Let assumption $\left(H_{0}\right)$ be satisfied. Let $u^{\varepsilon}$ be the solution to problem (1.1)-(1.2). Suppose that (3.3) and (3.8) with $\tau=0$ hold true. Let $K \subset M$ be $a$ compact subset, $y \in K, s>0$. Let $\phi:=\eta \zeta$ with $\eta$ and $\zeta$ as in Lemma 5.3. Then for every $0<\varepsilon<1$ inequality (1.22) holds true, for all $0<t_{0} \leq t<s, C_{3}, C_{4}$ being as in Lemma 5.7, and for some positive constant $C_{5}\left(t_{0}\right)$ independent of $\varepsilon$ and $(y, s)$. As a consequence, for all $0 \leq t_{0}<t<s$, inequality (1.24) holds true.

Proof. By (1.14),

$$
\int_{M} \frac{\phi}{s-t} \xi_{t}^{\varepsilon} d \mathcal{V}(x) \leq \frac{C_{5}}{\sqrt{s-t}},
$$

for some positive constant $C_{5}$, independent of $\varepsilon, y, s$. From (5.25) and (5.18) we can deduce (1.22). Thus (1.24) follows from Gronwall's inequality.

As a consequence, the next proposition gives uniform density bounds for the measures $\mu_{t}^{\varepsilon}$ at small scales. 
Proposition 5.9. Let assumption $\left(H_{0}\right)$ be satisfied. Let $u^{\varepsilon}$ be the solution to problem (1.1)-(1.2). Suppose that (3.3) and (3.8) with $\tau=0$ hold true. Then, for each compact subset $K \subset M$,

$\left(G_{1}^{\varepsilon}\right) \quad \int_{M} \phi_{y, s}(x, t) d \mu_{t}^{\varepsilon}(x) \leq \underline{C}$ for all $y \in K, 0<t_{0} \leq t<s$,

for some $\underline{C}=\underline{C}_{K}>0$, and

$\left(G_{2}^{\varepsilon}\right) \quad \mu_{t}^{\varepsilon}\left(B_{R}(x)\right) \leq \omega_{N-1} D_{0} R^{N-1} \quad$ for all $x \in K, 0<R<\tilde{R}, t \geq 0$,

for some $0<\tilde{R}<R_{0}$ and $D_{0}=D_{0}(\underline{C}, \tilde{R})>0$.

Proof. From (1.24) and (3.8) it easily follows $\left(G_{1}^{\varepsilon}\right)$. Observe that, for some $0<$ $\tilde{R}<R_{0}, C=C(\tilde{R})>0$, there holds, for all $x, y \in M, 0<R<\tilde{R}, t \geq 0$

$$
\frac{1}{R^{N-1}} \chi_{B_{R}(y)}(x) \leq C \phi_{y, s}(x, t),
$$

whenever $s-t=R^{2}$. This, combined with $\left(G_{1}^{\varepsilon}\right)$, yields $\left(G_{2}^{\varepsilon}\right)$.

\subsection{Further compactness properties}

Concerning the family $\left\{u^{\varepsilon}\right\}_{0<\varepsilon<1}$ of solutions to problem (1.1)-(1.2) we have the next compactness result.

Proposition 5.10. Let assumption $\left(H_{0}\right)$ be satisfied. Let $\left\{u^{\varepsilon}\right\}_{0<\varepsilon<1}$ be a family of solutions to problem (1.1)-(1.2) which is uniformly bounded, i.e. satisfying (3.3), and such that for each $T>0$ and for each compact set $K \subset M$

$$
\sup _{0<\varepsilon<1} \sup _{t \in[0, T]} \varepsilon \int_{K} E^{\varepsilon}(x, t) d \mathcal{V} \leq C,
$$

for some $C=C(K, T)>0$ independent of $\varepsilon$. Set $\mathcal{F}(s):=\int_{0}^{s} \sqrt{F(\tau)} d \tau$. Then there exist a subsequence $\left\{\mathcal{F}\left(u^{\varepsilon_{n}}\right)\right\} \subset\left\{\mathcal{F}\left(u^{\varepsilon}\right)\right\}$ and a function $v$ such that

$$
\mathcal{F}\left(u^{\varepsilon_{n}}\right) \rightarrow v \quad \text { as } n \rightarrow \infty,
$$

both in $C_{\mathrm{loc}}^{0, \alpha}\left([0, \infty) ; L_{\mathrm{loc}}^{1}(M)\right)$ for each $0 \leq \alpha<\frac{1}{2}$ and in $B V_{\mathrm{loc}}(M \times(0, \infty))$, and

$$
u^{\varepsilon_{n}} \rightarrow u^{*}:=\mathcal{F}^{-1}(v) \text { as } n \rightarrow \infty
$$

in $C_{\mathrm{loc}}^{0}\left([0, \infty) ; L_{\mathrm{loc}}^{1}(M)\right)$.

Moreover, $u^{*} \in L_{\mathrm{loc}}^{\infty}\left((0, \infty) ; B V_{\mathrm{loc}}(M)\right) \cap B V_{\mathrm{loc}}(M \times(0, \infty))$,

$$
\left|u^{*}\right|=1 \text { a.e. in } M \times(0, \infty),
$$

and the jump set of $u^{*}(\cdot, t)$ is locally $(N-1)$ - rectifiable for a.e. $t>0$. 
Proof. Let $T>0$ be fixed and let $\varphi \in C_{0}^{\infty}(M)$. By $\left(H_{0}\right)$ and (1.1)-(1.2), multiplying (1.1) by $\varphi^{2} \partial_{t} u^{\varepsilon}$ and integrating by parts we easily obtain

$$
\begin{aligned}
\int_{0}^{T} \int_{M} \varepsilon \varphi^{2}\left(\partial_{t} u^{\varepsilon}\right)^{2} d \mathcal{V} d t & \leq \int_{0}^{T} \int_{M} \varepsilon \varphi^{2}\left(\partial_{t} u^{\varepsilon}\right)^{2} d \mathcal{V} d t+\varepsilon \int_{M} \varphi^{2} E^{\varepsilon}(x, T) d \mathcal{V} \\
& \leq \varepsilon \int_{M} \varphi^{2} E^{\varepsilon}(x, 0) d \mathcal{V}-2 \varepsilon \int_{0}^{T} \int_{M} \varphi \partial_{t} u^{\varepsilon} \nabla u^{\varepsilon} \nabla \varphi d \mathcal{V} d t
\end{aligned}
$$

Since $\left|\nabla u^{\varepsilon}\right|^{2} \leq 2 E^{\varepsilon}(x, t)$, applying Young's inequality and (5.26) with $K=$ $\operatorname{supp} \varphi$ we easily obtain for any $\varphi \in C_{0}^{\infty}(M)$ the uniform bound

$$
\int_{0}^{T} \int_{M} \varepsilon \varphi^{2}\left(\partial_{t} u^{\varepsilon}\right)^{2} d \mathcal{V} d t \leq C .
$$

In addition, in view of (3.3) we also have

$$
\left|\mathcal{F}\left(u^{\varepsilon}\right)\right| \leq C \quad \text { in } M
$$

for every $\varepsilon>0$. From (5.26)-(5.30) and Young's inequality it follows that

$$
\left\|\mathcal{F}\left(u^{\varepsilon}\right)\right\|_{L^{\infty}\left((0, T) ; B V_{\mathrm{loc}}(M)\right)}+\left\|\partial_{t} \mathcal{F}\left(u^{\varepsilon}\right)\right\|_{L^{1}\left(0, T ; L_{\mathrm{loc}}^{1}(M)\right)} \leq C
$$

for every $\varepsilon>0$. As a consequence, $\mathcal{F}\left(u^{\varepsilon}\right)$ is $*$-weakly compact in $B V_{\text {loc }}(M \times$ $(0, \infty))$, since $T>0$ is arbitrary.

In view of (5.26), (5.30) and the estimate on the time derivative in (5.31) we can also infer that

$$
\left\|\mathcal{F}\left(u^{\varepsilon}\right)\right\|_{C^{0,1 / 2}\left([0, T] ; L_{\mathrm{loc}}^{1}(M)\right)} \leq C(T),
$$

for every $\varepsilon>0$. So, by Ascoli-Arzelà theorem, $\left\{\mathcal{F}\left(u^{\varepsilon}\right)\right\}_{\varepsilon>0}$ is compact in $C_{\text {loc }}^{0, \alpha}\left([0, \infty) ; L_{\text {loc }}^{1}(M)\right)$ for each $0 \leq \alpha<\frac{1}{2}$ and the same property with $\alpha=0$ clearly holds for $\left\{u^{\varepsilon}\right\}_{\varepsilon>0}$ by continuity of $\mathcal{F}^{-1}$. Thus, by a diagonal argument there exist a subsequence $\left\{\mathcal{F}\left(u^{\varepsilon_{n}}\right)\right\} \subset\left\{\mathcal{F}\left(u^{\varepsilon}\right)\right\}$ and a function $v$ such that (5.27)(5.28) holds. Moreover, it is direct to see from the uniform bound on the potential energy in (5.26) that $\left|u^{*}\right|=1$ a.e. in $M \times(0, \infty)$, and in turn that $u^{*} \in$ $L^{\infty}\left((0, \infty) ; B V_{\text {loc }}(M)\right) \cap B V_{\text {loc }}(M \times(0, \infty))$. Then the last statement follows from the rectifiability of the jump set for $B V$ functions.

\section{References}

[1] M. Alfaro, D. Hilhorst and H. Matano, The singular limit of the Allen-Cahn equation and the FitzHugh-Nagumo system, J. Differential Equations 245 (2008), 505-565. 
[2] D. Azagra, M. Jiménez-Sevilla and F. Macià, Generalized motion of level sets by functions of their curvatures on Riemannian manifolds, Calc. Var. Partial Differential Equations 33 (2008), 133-167.

[3] G. BARles, H. M. Soner and P. E. Souganidis, Front propagation and phase field theory, SIAM J. Control Optim. 31 (1993), 439-469.

[4] G. Bellettini and M. NovaGA, Comparison results between minimal barriers and viscosity solutions for geometric evolutions, Ann. Scuola Norm. Sup. Pisa Cl. Sci. (4) 26 (1998), 97-131.

[5] G. Bellettini and M. Paolini, Some results on minimal barriers in the sense of De Giorgi applied to driven motion by mean curvature, Rend. Acc. Naz. Sci. XL Mem. Mat. Appl. 19 (1995), 43-67.

[6] F. Bethuel, G. ORLAndi and D. Smets, Convergence of the parabolic GinzburgLandau equation to motion by mean curvature, Ann. of Math. 163 (2006), 37-163.

[7] K. A. BRAKKE, "The Motion of a Surface by its Mean Curvature", Princeton University Press, Princeton, NJ, 1978.

[8] L. BRONSARD and R. V. KoHn, On the slowness of phase boundary motion in one space dimension, preprint 13, Lefschetz Center, Brown University, 1989.

[9] L. BRONSARD and R. V. KoHN, Motion by mean curvature as the singular limit of Ginzburg-Landau dynamics, J. Differential Equations 90 (1991), 211-237.

[10] J. CARR and R. PEGo, Very slow phase separation in one dimension, In: "Proc. Conf. on Phase Transitions", M. Rasele et al. (eds.), Lect. Notes in Phys., Vol. 344, Springer, 1989, 216-226.

[11] J. CARR and R. PEgo, Metastable patterns in solutions of $u_{t}=\varepsilon^{2} u_{x x}-f(u)$, Comm. Pure Appl. Math. 42 (1989), 523-576.

[12] X. Chen, Generation and propagation of the interface for reaction-diffusion equations, J. Differential Equations 96 (1992), 116-141.

[13] Y. G. Chen, Y. Giga and S. Goto, Uniqueness and existence of viscosity solutions of generalized mean curvature flow equation, J. Differential Geom. 33 (1991), 749-786.

[14] P. DE Mottoni and M. Schatzman, Evolution geometrique d'interfaces, C. R. Acad. Sci. Paris 309 (1989), 453-458.

[15] P. De Mottoni and M. Schatzman, Geometrical evolution of developed interfaces, Trans. Amer. Math. Soc. 347 (1995), 1533-1589.

[16] M. DEL PINO, M. KowALCZYK, J. WEI and J. YANG, Interface foliation near minimal submanifolds in Riemannian manifolds with positive Ricci curvature, Geom. Funct. Anal. 20 (2010), 918-957.

[17] J. DoDZIUK, Maximum principle for parabolic inequalities and the heat ow on open manifolds, Indiana Univ. Math. J. 32 (1983), 703-716.

[18] K. ECKER, A local monotonicity formula for mean curvature flow. Ann. of Math. (2) 154 (2001), 503-525.

[19] L. C. Evans and J. SpRuCK, Motion of level sets by mean curvature, I, J. Differential Geom. 33 (1991), 635-681.

[20] L. C. Evans and J. SPRUCK, Motion of level sets by mean curvature, II, Trans. Amer. Math. Soc. 330, (1992), 321-332.

[21] L. C. EvAnS and J. SPRUCK, Motion of level sets by mean curvature, III, J. Geom. Anal. 2 (1992), 121-150.

[22] L. C. Evans and J. SpruCK, Motion of level sets by mean curvature, IV, J. Geom. Anal. 5 (1995), 77-114.

[23] L. C. Evans, H. M. Soner and P. E. Souganidis, Phase transitions and generalized motion by mean curvature, Comm. Pure Appl. Math. 45 (1992), 1097-1123.

[24] P. C. FIFE, "Dynamics of Internal Layers and Diffusive Interfaces", CMBS-NSF Ref. Conf. Sci. Appl. Math., Vol. 53, 1988. 
[25] G. Fusco, A geometric approach to the dynamics of $u_{t}=\varepsilon^{2} u_{x x}+f(u)$ for small $\varepsilon$, In: "Proc. Conf. Stuttgart" 1988, K. Kirchgassner (ed.), Lecture Notes in Physics, Vol. 359, 1990, 53-73.

[26] S. Gallot, D. Hulin and J. Lafontaine, "Riemannian Geometry", Universitext, Springer, 1993.

[27] G. HUISKEN, Evolution of hypersurfaces by their curvature in Riemannian manifolds, Doc. Math. Extra Vol. ICM II (1998), 349-360.

[28] G. Huisken, Contracting convex hypersurfaces in Riemannian manifolds by their mean curvature, Invent. Math. 84 (1986), 463-480.

[29] J. E. Hutchinson and Y. TONEGAWA, Convergence of phase interfaces in the van der Waals-Cahn-Hilliard theory, Calc. Var. Partial Differential Equations 10 (2000), 49-84.

[30] T. ILMANEN, Generalized motion of sets by mean curvature on a manifold, Indiana Univ. Math. J. 41 (1992), 671-705.

[31] T. Ilmanen, Convergence of the Allen-Cahn equation to the Brakke's motion by mean curvature, J. Differential Geom. 31 (1993), 417-461.

[32] T. Ilmanen, "Elliptic Regularization and Partial Regularity for Motion by Mean Curvature", Mem. Americ. Math. Soc., Vol. 520, 1994.

[33] R. L. JERRARD and H. M. SONER, Scaling limits and regularity results for a class of Ginzburg-Landau systems, Ann. Inst. H. Poincaré Anal. Non Linéaire 16 (1999), 423-466.

[34] J. JosT, "Riemannian Geometry and Geometric Analysis", Universitext, Springer-Verlag, 2005.

[35] L. ModicA, A gradient bound and a Liouville theorem for nonlinear Poisson equations, Comm. Pure Appl. Math. 38 (1985), 679-684.

[36] L. MODICA, The gradient theory of phase transitions and the minimal interface criterion Arch. Ration. Mech. Anal. 98 (1986), 123-142 .

[37] M. NovaGA, Soluzioni di tipo barriera (Italian), Boll. Unione Mat. Ital. 8 (2001), 131142.

[38] J. Rubinstein, P. Sternberg and J. B. Keller, Fast reaction, slow diffusion and curve- shortening, SIAM J. Appl. Math. 49 (1989), 116-133.

[39] F. PACARD and M. RITORÉ, From constant mean curvature hypersurfaces to the gradient theory of phase transitions, J. Differential Geom. 64 (2003), 359-423.

[40] A. Pis Ante and M. Ponsiglione, Phase transitions and minimal hypersurfaces in hyperbolic space, Comm. Partial Differential Equations 36 (2011), 819-849.

[41] A. PisAnTE and F. Punzo, Allen-Cahn approximation of mean curvature flow in Riemannian manifolds II, Brakke's flows, Comm. Cont. Math. 17 (2015), 35 pages.

[42] N. SATO, A simple proof of convergence of the Allen-Cahn equation to Brakke's motion by mean curvature, Indiana Univ. Math. J. 57 (2008), 1743-1751.

[43] H. M. SONER, Ginzburg-Landau equation and motion by mean curvature, I: Convergence, J. Geom. Anal. 7 (1997), 437-475.

Dipartimento di Matematica "G. Castelnuovo" "Sapienza" Università di Roma

P.le A. Moro, 5

00185 Roma, Italia

pisante@mat.uniroma1.it

Dipartimento di Matematica e Informatica

Università della Calabria

Via P. Bucci, 31b

87036 Rende (CS), Italia

fabio.punzo@unical.it 